Journal of Mathematical Physics, Analysis, Geometry

2016, vol. 12, No. 1, pp. 17-47

\title{
Transformation Operators and Modified Sobolev Spaces in Controllability Problems on a Half-Axis
}

\author{
L.V. Fardigola \\ B. Verkin Institute for Low Temperature Physics and Engineering \\ National Academy of Sciences of Ukraine \\ 47 Nauki Ave., Kharkiv, 61103, Ukraine \\ E-mail: fardigola@ukr.net,fardigola@ilt.kharkov.ua
}

Received September 20, 2015,

published online December 4, 2015

In the paper, the control system $w_{t t}=\frac{1}{\rho}\left(k w_{x}\right)_{x}+\gamma w, w_{x}(0, t)=u(t)$, $x>0, t \in(0, T)$, is considered in special modified spaces of Sobolev type. Here $\rho, k$, and $\gamma$ are given functions on $[0,+\infty) ; u \in L^{\infty}(0, \infty)$ is a control; $T>0$ is a constant. The growth of distributions from these spaces depends on the growth of $\rho$ and $k$. With the aid of some transformation operators, it is proved that the control system replicates the controllability properties of the auxiliary system $z_{t t}=z_{\xi \xi}-q^{2} z, z_{\xi}(0, t)=v(t), \xi>0, t \in(0, T)$, and vise versa. Here $q \geq 0$ is a constant and $v \in L^{\infty}(0, \infty)$ is a control. For the main system, necessary and sufficient conditions of the $L^{\infty}$-controllability and the approximate $L^{\infty}$-controllability are obtained from those known for the auxiliary system.

Key words: wave equation, half-axis, controllability problem, transformation operator, modified space of Sobolev type.

Mathematics Subject Classification 2010: 93B05, 35B30, 35L05.

\section{Introduction}

Controllability problems for hyperbolic equations with constant and variable coefficients were studied in a number of recent papers, e.g., [2-4, 8-18, 22, 23, 27-29, 31, 33-38, 40] and many others. However, controllability problems for a distributed parameter system on domains unbounded with respect to the space variables have not been investigated fully. Nevertheless, these problems were studied for the wave equation in $\mathbb{R}^{3}$ in [3], on a half-plane in $[9,16,17]$, and on a half-axis in [10-15, 27-29, 36].

(C) L.V. Fardigola, 2016 
In the present paper, we study the wave equation with variable coefficients

$$
w_{t t}=\frac{1}{\rho}\left(k w_{x}\right)_{x}+\gamma w, \quad x>0, t \in(0, T),
$$

controlled by the Neumann boundary condition

$$
w_{x}(0, \cdot)=u \quad \text { on }(0, T)
$$

under the initial conditions

$$
w(\cdot, 0)=w_{0}^{0}, \quad w_{t}(\cdot, 0)=w_{1}^{0} \quad \text { on }(0,+\infty),
$$

where $T>0$ is a constant; $\rho, k, \gamma, w_{0}^{0}$, and $w_{1}^{0}$ are given functions; $u \in L^{\infty}(0, T)$ is a control. In addition, we assume $\rho, k \in C^{1}[0,+\infty)$ are positive on $[0,+\infty)$, $(\rho k) \in C^{2}[0,+\infty),(\rho k)^{\prime}(0)=0$, and

$$
\sigma(x)=\int_{0}^{x} \sqrt{\rho(\mu) / k(\mu)} d \mu \rightarrow+\infty \quad \text { as } x \rightarrow+\infty .
$$

Moreover, we assume

$$
P(k, \rho)-\gamma \in L^{\infty}(0,+\infty) \bigcap C^{1}[0,+\infty)
$$

and

$$
\exists q=\text { const } \geq 0 \quad \sigma \sqrt{\frac{\rho}{k}}\left(P(k, \rho)-\gamma-q^{2}\right) \in L^{1}(0,+\infty),
$$

where $P(k, \rho)=\frac{1}{4} \sqrt{\frac{k}{\rho}}\left(\sqrt{\frac{k}{\rho}}\left(\frac{k^{\prime}}{k}+\frac{\rho^{\prime}}{\rho}\right)\right)^{\prime}+\left(\frac{1}{4} \sqrt{\frac{k}{\rho}}\left(\frac{k^{\prime}}{k}+\frac{\rho^{\prime}}{\rho}\right)\right)^{2}$. This control system is considered in modified Sobolev spaces (see Sec. 2).

Note that (1.1) can be reduced to a wave equation with constant coefficients and a constant potential by using transformation operators. Various transformation operators were used for studying different PDE's in [14-17, 24-30, 32] and others. Note that the application of transformation operators is a key point of the paper.

Let us recall the results obtained in $[10-14,27-29,36]$ for (1.1)-(1.3) with $\rho=k=$ const, i. e., for the wave equation

$$
y_{t t}=y_{\lambda \lambda}-\left(q^{2}+r\right) y, \quad \lambda>0, t \in(0, T),
$$

controlled by the Dirichlet boundary condition

$$
y(0, \cdot)=v \quad \text { on }(0, T)
$$


or the Neumann boundary condition

$$
y_{\lambda}(0, \cdot)=v \quad \text { on }(0, T) .
$$

Here $q \geq 0$ is a given constant, $r \in C^{1}[0,+\infty)$ is a given function such that $x r \in L^{1}(0,+\infty)$, and $v \in L^{\infty}(0, T)$ is a control. This system is considered in Sobolev spaces (see Sec. 2).

For control problem (1.7), (1.8), the approximate $L^{\infty}$-controllability and the $L^{\infty}$-controllability were studied at a given time and at a free time for $r=q=0$ in [36], and for $r=0$ in [12]. In this case $(r=0)$, the solutions to (1.7), (1.8) were found in explicit form, and necessary and sufficient conditions for the (approximate) $L^{\infty}$-controllability were obtained. In [12], it was proved that controllability properties of (1.7), (1.8) with $r=0$ are similar at a given time for the cases $q=0$ and $q>0$. Therein, it was also proved that the case $q=0$ essentially differs from the case $q>0$ at a free time. In particular, if $q>0$, then each initial state of control system (1.7), (1.8) with $r=0$ is approximately $L^{\infty}$-controllable at a free time. But, if $q=0$, then the initial state of this system is approximately $L^{\infty}$-controllable at a free time iff $y_{t}(\cdot, 0)=y_{\lambda}(\cdot, 0)$. The results of [12] were used in [14].

In [14], control system (1.7), (1.8) was studied under the addition restriction $|r(\lambda)| \leq \alpha e^{-\lambda}, \lambda>0$, by using the well-known transformation operator (see, e.g., [32, Chap. 3]) of the Sturm-Liouville problem saving the asymptotics of solutions at infinity. This operator and its inverse were extended from $L^{2}(0,+\infty)$ to the Sobolev spaces $\widetilde{H}_{0}^{m}, m=\overline{-2,2}$, in [14]. Here $\widetilde{H}_{0}^{m}$ is the subspace of all odd distributions in $H_{0}^{m}, m=\overline{-2,2}$ (see Sec. 2). This extended transformation operator was studied and its properties were obtained in [14]. In particular, this operator is an automorphism of $\widetilde{H}_{0}^{m}, m=\overline{-2,2}$. Using the extended transformation operator, it was shown that control system (1.7), (1.8) with exponentially perturbed potential $q^{2}$ replicated the controllability properties of its original control system of the same type with the constant potential $q^{2}(r=0)$ and vise versa (see [14]). In particular, necessary and sufficient conditions for the approximate $L^{\infty}$-controllability and the $L^{\infty}$-controllability of (1.7), (1.8) with the exponential perturbed potential $q^{2}$ were obtained at a given time and at a free time. A bit later, in [29], sufficient conditions of the (approximate) $L^{\infty}$-controllability were proved at a given time and at a free time for (1.7), (1.8) in the case $q=0$ and a general $r$. Therein, it was announced that the main properties obtained in [14] for the transformation operator and its inverse are true for $r$ without the exponential restriction. Thus, all results of [14] are still valid, but without this exponential restriction.

For control system (1.7), (1.9), the approximate $L^{\infty}$-controllability and the $L^{\infty}$-controllability were studied at a given time (for $q \geq 0$ and $r=0$ in [10], for $q=0$ and $r \neq 0$ in [27]) and at a free time (for $q=r=0$ in [11], for $q \geq 0$ and 
$r=0$ in [13], for $q=0$ and $r \neq 0$ in [28]). In [28], the transformation operator of the Sturm-Liouville problem saving the asymptotics of solutions at infinity and its inverse were extended from $L^{2}(0,+\infty)$ to the Sobolev spaces $\widehat{H}_{0}^{m}, m=$ $\overline{-1,1}$. Here $\widehat{H}_{0}^{m}$ is the subspace of all even distributions in $H_{0}^{m}, m=\overline{-1,1}$ (see Sec. 2). This extended transformation operator was studied and its properties were obtained in [28]. In particular, this operator is an automorphism of $\widehat{H}_{0}^{m}$, $m=\overline{-1,1}$. Using the properties of the extended transformation operator, only sufficient conditions of the (approximate) $L^{\infty}$-controllability were proved at a given time and at a free time for (1.7), (1.9) for the case $q=0$ and $r \neq 0$ in [28].

Note that if $\operatorname{supp} r$ is bounded, the transformation operator of the SturmLiouville problem saving the initial data of solutions was used to study the (approximate) $L^{\infty}$-controllability of (1.7) with $q=0$ only at a given time in [27].

In [15], the approximate $L^{\infty}$-controllability and the $L^{\infty}$-controllability for equation (1.1) controlled by the Dirichlet boundary condition were studied. Note that the Sobolev spaces $H_{0}^{s}, m \in \mathbb{R}$, are the natural "environment" for the solutions to hyperbolic equations with constant coefficients, in particular, to Eq. (1.7) (see, e.g., [21]). Evidently, the growth of solutions to equations with variable coefficients depends on the properties of these coefficients at infinity. To study equation (1.1) with the Dirichlet boundary control, new transformation operators were introduced and investigated in [15]. They transform the solutions to (1.7), (1.8) with $r=0$ and some $q$ into the solutions to (1.1) with the Dirichlet boundary control. Together with one of these operators, special modified spaces of Sobolev type were introduced where Eq. (1.7) was considered. Using these operators, it was proved that Eq. (1.1) controlled by the Dirichlet boundary condition replicated the controllability properties of control system (1.7), (1.8) with $r=0$ and some $q$. In particular, necessary and sufficient conditions for the approximate $L^{\infty}$-controllability and the $L^{\infty}$-controllability were obtained at a given time and at a free time for (1.1) with the Dirichlet boundary control.

A similar approach is used in the present paper. To study control system (1.1), (1.3), we apply the composition $\mathbf{S} \mathbf{T}_{r}$ of two operators transforming the solutions to (1.7), (1.9) with $r=0$ and $q$ defined by (1.6) to the solutions to (1.1), (1.2). The first of them is the operator $\mathbf{S}$ transforming the solutions to (1.7), (1.9) with $q$ and $r$ defined by (1.6) and (3.3) to the solutions to (1.1), (1.2). This operator was introduced and studied in [15]. Together with the operator $\mathbf{S}$, there were introduced and studied special modified spaces $\mathbb{H}^{m}, m=\overline{-2,2}$, of Sobolev type where the space $L^{2}(\mathbb{R})$ is replaced by the space $L_{\rho}^{2}(\mathbb{R})$ with the weight $\sqrt{\hat{\rho}}$, and the differential operator $d / d x$ is replaced by the "linearly deformed" one, $\sqrt{\widehat{k} / \widehat{\rho}}\left(d / d x+\left(\widehat{\rho}^{\prime} / \widehat{\rho}+\widehat{k}^{\prime} / \widehat{k}\right) / 4\right), \widehat{k}$ and $\widehat{\rho}$ are the even extensions of $k$ and $\rho$, respectively (see Sec. 2). The operator $\mathbf{S}$ is isometric from $H_{0}^{m}$ into $\mathbb{H}^{m}$, in particular, $\mathbf{S}\left(H_{0}^{m}\right)=\mathbb{H}^{m}, m=\overline{-2,2}$. The operator $\mathbf{S}$ saves the 
value of functions at the origin, but it does not save their asymptotics at infinity. The growth of distribution from $\mathbb{H}^{m}$ is associated with the data $\rho$ and $k$ of equation (1.1), $m=\overline{-2,2}$. The second of these two operators is the operator $\mathbf{T}_{r}$ transforming the solutions to (1.7), (1.9) with $r=0$ and $q$ defined by (1.6) to the solutions to (1.7), (1.9) with the same $q$ and $r$ defined by (3.3). This operator was studied in [28]. The operator $\mathbf{T}_{r}$ and its inverse are bounded from $H_{0}^{m}$ to $H_{0}^{m}$ and $\mathbf{T}_{r}\left(\widehat{H}_{0}^{m}\right)=\widehat{H}_{0}^{m}, m=\overline{-1,1}$. It saves the asymptotics of functions at infinity, but it does not save their values at the origin. The operators $\mathbf{S}, \mathbf{T}_{r}$ and the spaces $\mathbb{H}^{m}, m=\overline{-1,1}$, associated with the operator $\mathbf{S}$, are the maim tools of this paper. Using the operator $\mathbf{S T}_{r}$, we conclude that control system (1.1), (1.2) replicates the controllability properties of control system (1.7), (1.9) with $r=0$ and $q$ determined by (1.6) (Corollaries 5.1, 5.6). In particular, we obtain necessary and sufficient conditions of the approximate $L^{\infty}$-controllability and the $L^{\infty}$-controllability at a given time and at a free time for (1.1), (1.2). If $q>0$, then each initial state of control system (1.1), (1.2) is approximately $L^{\infty}$-controllable at a free time (Theorem 5.8). But, if $q=0$, then an initial state of this system is approximately $L^{\infty}$-controllable at a free time iff its coordinates (i. e., $w(x, 0)$ and $\left.w_{t}(x, 0)\right)$ are related (Theorem 5.7). The similar relation is necessary for the $L^{\infty}$-controllability and the approximate $L^{\infty}$-controllability at a given time for both cases: $q=0$ and $q>0$ (Theorem 5.5), i. e., for each time $T>0$ we have the set of admissible initial states described by (5.4) and (5.5).

Note that the main results of [28] are particular cases of the results of the present paper. Moreover, according to Corollary 5.7, sufficient conditions from [28, Theorems 3.6 and 3.7] are also necessary for the approximate $L^{\infty}$-controllability of (1.7), (1.9) with $q=0$ at a free time.

Thus the application of the transformation operators $\mathbf{S}$ and $\mathbf{T}_{R}$ and the modified Sobolev spaces $\mathbb{H}^{m}, m=\overline{-1,1}$, is the focal point in the study of the $L^{\infty}$ controllability problems for equation (1.1) with variable coefficients.

In Secs. 2 and 3, the definitions of the spaces and the operators used in the paper are given and their properties are studied. In addition, problem (1.1)-(1.3) is reduced to $(2.3),(2.4)$.

In Sec. 1, some transformation between the solutions to control systems (2.3), (2.4) and (2.6), (2.7) are performed.

In Sec. 2, necessary and sufficient conditions for the approximate $L^{\infty}$-controllability and the $L^{\infty}$-controllability are obtained at a given time and at a free time.

In Sec. 6, some examples illustrating the results of Secs. 3-5 are considered.

In Sec. 7, some auxiliary assertion is proved. 


\section{Notation}

Let us give the definitions of the spaces used in the paper. Let $\mathcal{D}$ be the space of infinitely differentiable functions with compact supports, where $\varphi_{n} \rightarrow 0$ as $n \rightarrow \infty$ iff there exists $a>0$ such that for each $n=\overline{1, \infty}$ we have $\operatorname{supp} \varphi_{n} \in$ $[-a, a]$, and for each $m=\overline{1, \infty}$ we have $\left\|\varphi_{n}^{(m)}\right\|_{L^{\infty}[-a, a]} \rightarrow 0$ as $n \rightarrow \infty$. Let $\mathcal{D}^{\prime}$ be the dual space. Let also $\mathcal{S}$ be the Schwartz space of rapidly decreasing functions on $\mathbb{R}$, and $\mathcal{S}^{\prime}$ be the dual space of tempered distributions.

By $H_{l}^{s}(s, l \in \mathbb{R})$, denote the Sobolev spaces:

$$
\begin{aligned}
H_{l}^{s} & =\left\{\varphi \in \mathcal{S}^{\prime} \mid\left(1+|D|^{2}\right)^{s / 2}\left(1+|x|^{2}\right)^{l / 2} \varphi \in L^{2}(\mathbb{R})\right\} \\
\|\varphi\|_{l}^{s} & =\left(\int_{-\infty}^{\infty}\left|\left(1+|D|^{2}\right)^{s / 2}\left(1+|x|^{2}\right)^{l / 2} \varphi(x)\right|^{2} d x\right)^{1 / 2},
\end{aligned}
$$

where $D=-i \partial / \partial x,|\cdot|$ is the Euclidean norm. In particular, we have $H_{0}^{0}=L^{2}(\mathbb{R})$, $\|\cdot\|_{0}^{0}=\|\cdot\|_{L^{2}(\mathbb{R})}$. For a positive integer $p$, we have $H_{0}^{p}=\left\{\varphi \in L_{\text {loc }}^{2}(\mathbb{R}) \mid \forall m=\right.$ $\left.\overline{0, p} \varphi^{(m)} \in H_{0}^{0}\right\},\|\varphi\|_{0}^{p}=\left(\sum_{m=0}^{p}\left(\left\|\varphi^{(m)}\right\|_{0}^{0}\right)^{2}\right)^{1 / 2}$, and $H_{0}^{-p}=\left(H_{0}^{p}\right)^{\prime}$. It is well known [21, Chap. 1] that

$$
\|\varphi\|_{l}^{s} \leq\|\varphi\|_{l^{\prime}}^{s^{\prime}}, \quad s \leq s^{\prime}, l \leq l^{\prime}, \varphi \in H_{l^{\prime}}^{s^{\prime}} .
$$

Therefore, $H_{l}^{s} \supset H_{l^{\prime}}^{s^{\prime}}$ is a continuous embedding, $s \leq s^{\prime}, l \leq l^{\prime}$.

A distribution $f \in \mathcal{D}^{\prime}$ is said to be odd if $\langle f, \varphi(\xi)\rangle=-\langle f, \varphi(-\xi)\rangle, \varphi \in \mathcal{D}$, and it is said to be even if $\langle f, \varphi(\xi)\rangle=\langle f, \varphi(-\xi)\rangle, \varphi \in \mathcal{D}$. Here $\langle f, \varphi\rangle$ is the value of the distribution $f \in \mathcal{D}^{\prime}$ on the test function $\varphi \in \mathcal{D}$. By $\widetilde{H}_{l}^{s}$ and $\widehat{H}_{l}^{s}$, denote the subspaces of odd and even, respectively, distributions in $H_{l}^{s}$. Denote also $\widehat{\mathbf{H}}^{1}=\widehat{H}_{0}^{1} \times \widehat{H}_{0}^{0}$ with the norm $\|\cdot\| \|^{0}$.

By $\widehat{\rho}, \widehat{k}$, and $\widehat{\gamma}$, denote the even extension of $\rho, k$, and $\gamma$, respectively. Denote $\eta=(\widehat{k} \hat{\rho})^{1 / 4}, \theta=(\widehat{k} / \widehat{\rho})^{1 / 4}$, and $\mathcal{D}_{\eta \theta}=\theta^{2}\left(d / d x+\eta^{\prime} / \eta\right)=\sqrt{\widehat{k} / \widehat{\rho}}\left(d / d x+\left(\widehat{\rho}^{\prime} / \widehat{\rho}+\right.\right.$ $\left.\left.\widehat{k}^{\prime} / \widehat{k}\right) / 4\right)$. We see that $\eta \in C^{2}(\mathbb{R})$ and $\theta \in C^{1}(\mathbb{R})$.

Further throughout the section, we will assume $p=0,1$.

Introduce the space

$$
\mathbb{H}^{p}=\left\{\varphi \in L_{\mathrm{loc}}^{2}(\mathbb{R}) \mid \forall m=\overline{0, p}\left(\frac{\eta}{\theta}\left(\mathcal{D}_{\eta \theta}^{m} \varphi\right)\right) \in H_{0}^{0}\right\}
$$

with the norm

$$
\llbracket \varphi \rrbracket^{p}=\left(\sum_{m=0}^{p}\left(\left\|\frac{\eta}{\theta}\left(\mathcal{D}_{\eta \theta}^{m} \varphi\right)\right\|_{0}^{0}\right)^{2}\right)^{1 / 2}, \quad \varphi \in \mathbb{H}^{p},
$$


and the dual space $\mathbb{H}^{-p}=\left(\mathbb{H}^{p}\right)^{\prime}$ with the norm $\llbracket f \rrbracket^{-p}=\sup \left\{|\langle f, \varphi\rangle\rangle\left|/ \llbracket \varphi \rrbracket^{p}\right|\right.$ $\left.\llbracket \varphi \rrbracket^{p} \neq 0\right\}$, where $\langle\langle f, \varphi\rangle\rangle$ is the value of the distribution $f \in \mathbb{H}^{-p}$ on the test function $\varphi \in \mathbb{H}^{p}$. In particular, we have $\mathbb{H}^{0}=\left(\mathbb{H}^{0}\right)^{\prime}$ and $\langle\langle f, \varphi\rangle\rangle=\left\langle\frac{\eta}{\theta} f, \frac{\eta}{\theta} \varphi\right\rangle=$ $\int_{-\infty}^{\infty} \sqrt{\widehat{\rho}(x)} f(x) \varphi(x) d x, f, \varphi \in \mathbb{H}^{0}$. Put, $\left\langle\left\langle\mathcal{D}_{\eta \theta} f, \varphi\right\rangle\right\rangle=-\left\langle\left\langle f, \mathcal{D}_{\eta \theta} \varphi\right\rangle\right\rangle, f \in \mathbb{H}^{-p}$, $\varphi \in \mathbb{H}^{p+1}, p \neq 2$. The spaces $\mathbb{H}^{p}$ and $\mathbb{H}^{-p}$ are studied in Section 3.. In particular, it is proved that $\mathcal{D} \subset \mathbb{H}^{p} \subset \mathbb{H}^{-p} \subset \mathcal{D}^{\prime}$ are continuous embeddings [15].

Introduce also the spaces

$$
\begin{aligned}
& \mathcal{H}^{0}=\left\{\varphi \in L_{\mathrm{loc}}^{2}(0,+\infty) \mid\left(\frac{\eta}{\theta} \varphi\right) \in L^{2}(0,+\infty)\right\}, \\
& \mathcal{H}^{1}=\left\{\varphi \in \mathcal{H}^{0} \mid\left(\frac{\eta}{\theta} \mathcal{D}_{\eta \theta} \varphi\right) \in \mathcal{H}^{0}\right\}
\end{aligned}
$$

with the norm

$$
][\varphi]]^{p}=\left(\sum_{m=0}^{p}\left(\left\|\frac{\eta}{\theta}\left(\mathcal{D}_{\eta \theta}^{m} \varphi\right)\right\|_{L^{2}(0,+\infty)}\right)^{2}\right)^{1 / 2}, \quad \varphi \in \mathbb{H}^{p},
$$

and the dual space $\mathcal{H}^{-p}=\left(\mathcal{H}^{p}\right)^{\prime}$ with the norm of the conjoint space $\left.][f]\right]^{-p}=$ $\left.\left.\left.\sup \{|\langle[f, \varphi]\rangle| /][\varphi]]^{p} \mid\right][\varphi]\right]^{p} \neq 0\right\}$, where $\langle[f, \varphi]\rangle$ is the value of the distribution $f \in \mathcal{H}^{-p}$ on the test function $\varphi \in \mathcal{H}^{p}$. In particular, we have $\mathcal{H}^{0}=\left(\mathcal{H}^{0}\right)^{\prime}$ and $\langle[f, \varphi]\rangle=\int_{0}^{\infty}\left(\frac{\eta(x)}{\theta(x)}\right)^{2} f(x) \varphi(x) d x=\int_{0}^{\infty} \sqrt{\rho(x)} f(x) \varphi(x) d x, f, \varphi \in \mathcal{H}^{0}$.

We can see that the restriction of an even function from $\mathbb{H}^{p}$ to $[0,+\infty)$ belongs to $\mathcal{H}^{p}$ and vise versa: the even extension of a function from $\mathcal{H}^{p}$ belongs to $\mathbb{H}^{p}$. Therefore the restriction of an even distribution from $\mathbb{H}^{-p}$ to $\mathcal{H}^{p}$ belongs to $\mathcal{H}^{-p}$ and vise versa: the even extension of a distribution from $\mathcal{H}^{-p}$ belongs to $\mathbb{H}^{-p}$.

By $\widehat{\mathbb{H}}^{m}$, denote the subspace of even distributions in $\mathbb{H}^{m}, m=\overline{-1,1}$, and denote $\mathbb{H}^{1}=\widehat{\mathbb{H}}^{1} \times \widehat{\mathbb{H}}^{0}$ with the norm $\left[\llbracket \cdot \|^{0}\right.$.

We treat (1.2) as the value of the distribution $w$ at the point $x=0$ in $\mathcal{D}^{\prime}$ (see [1, Chap.1] or [14]). We say that a distribution $f \in \mathcal{H}^{-p}$ has the value $f_{0} \in \mathbb{R}$ at the point $x=0\left(f(0)=f_{0}\right)$ iff for any $\varphi \in \mathcal{D}, \operatorname{supp} \varphi \in[0,+\infty)$, we have $\left\langle f_{\alpha}, \varphi\right\rangle \rightarrow\left\langle f_{0}, \varphi\right\rangle$ as $\alpha \rightarrow+0$. Here $f_{\alpha}(x)=f(\alpha x)$, i.e., $\left\langle f_{\alpha}, \varphi\right\rangle=\left\langle f, \frac{1}{\alpha} \varphi_{1 / \alpha}\right\rangle$, $\varphi_{1 / \alpha}(x)=\varphi(x / \alpha), x \in \mathbb{R}$.

We consider control system (1.1)-(1.3) in the spaces $\mathcal{H}^{-m}, m=0$, 1, i.e., $\left(\frac{d}{d t}\right)^{m} w:[0, T] \rightarrow \mathcal{H}^{-m+1}, m=0,1,2, w_{0}^{0} \in \mathcal{H}^{1}$, and $w_{1}^{0} \in \mathcal{H}^{0}$. Evidently, Eq. (1.1) can be rewritten in the form

$$
w_{t t}=\mathcal{D}_{\eta \theta}^{2} w+(\gamma-\nu) w, \quad x>0, t \in(0, T),
$$

where $\nu=\mathcal{D}_{\eta \theta}\left(\theta^{2} \eta^{\prime} / \eta\right)$.

Let $w \in \mathcal{H}^{0}$ be a solution to control system (2.2), (1.2), (1.3). By $W, W_{0}^{0}$, and $W_{1}^{0}$, denote the even extension of $w, w_{0}^{0}$, and $w_{1}^{0}$ with respect to $x$, respectively. 
Taking into account Lemma 7.1, we see that

$$
\begin{aligned}
& W_{t t}=\mathcal{D}_{\eta \theta}^{2} W+(\widehat{\gamma}-\nu) W-2 \eta^{2}(0) u \delta, \quad x \in \mathbb{R}, t \in(0, T), \\
& W(\cdot, 0)=W_{0}^{0}, \quad W_{t}(\cdot, 0)=W_{1}^{0} \quad \text { on } \mathbb{R},
\end{aligned}
$$

where $\left(\frac{d}{d t}\right)^{m} W:[0, T] \rightarrow \widehat{\mathbb{H}}^{-m+1}, m=0,1,2, W_{0}^{0} \in \widehat{\mathbb{H}}^{1}, W_{1}^{0} \in \widehat{\mathbb{H}}^{0}, \delta$ is the Dirac distribution with respect to $x$. Let $W$ be a solution to (2.3), (2.4) and $W^{+}$be its restriction to $[0,+\infty) \times[0, T]$. According to Corollary 4.3 (see below Section 4.), we have

$$
\mathcal{D}_{\eta \theta} W^{+}(0, \cdot)=u \quad \text { on }(0, T) .
$$

Therefore, $W^{+}$is a solution to $(2.2),(1.2),(1.3)$.

Together with control system (2.3), (2.4) with a general wave operator, consider the auxiliary control system with the simplest wave operator

$$
\begin{aligned}
& Z_{t t}=Z_{\xi \xi}-q^{2} Z-2 v \delta, \quad \xi \in \mathbb{R}, t \in(0, T), \\
& Z(\cdot, 0)=Z_{0}^{0}, \quad Z_{t}(\cdot, 0)=Z_{1}^{0} \quad \text { on } \mathbb{R},
\end{aligned}
$$

where $\left(\frac{d}{d t}\right)^{m} Z:[0, T] \rightarrow H_{0}^{-m+1}, m=0,1,2, Z_{0}^{0} \in \widehat{H}_{0}^{1}, Z_{1}^{0} \in \widehat{H}_{0}^{0}, \delta$ is the Dirac distribution with respect to $\xi, v \in L^{\infty}(0, T)$ is a control, $q$ is the constant from condition (1.6). Let $Z$ be a solution to $(2.6),(2.7)$ and $Z^{+}$be its restriction to $[0,+\infty) \times[0, T]$. It is proved (see Theorem 4.1 below) that

$$
\left(Z^{+}\right)_{x}(0, \cdot)=v \quad \text { on }(0, T) .
$$

To formulate controllability properties of control system $(2.6),(2.7)$, recall some definitions and assertions from [12]. Let $\beta>0, \boldsymbol{\Phi}_{\beta}: \mathcal{S}^{\prime} \rightarrow \mathcal{S}^{\prime}, D\left(\boldsymbol{\Phi}_{\beta}\right)=$ $\left\{g \in \mathcal{S}^{\prime} \mid g\right.$ is odd and $\left.\operatorname{supp} g \subset[-\beta, \beta]\right\}$,

$$
\boldsymbol{\Phi}_{\beta} g=\mathcal{F}_{\sigma \rightarrow \xi}^{-1}\left(\frac{-i}{\sqrt{\sigma^{2}+q^{2}}}(\mathcal{F} g)\left(\sqrt{\sigma^{2}+q^{2}}\right)\right), \quad g \in D\left(\mathbf{\Phi}_{\beta}\right),
$$

where $\mathcal{F}$ is the Fourier transform operator. It is evident if $q=0$, then $\boldsymbol{\Phi}_{\beta}=\mathrm{Id}$ (where Id is the identity operator). In particular,

$$
\left(\mathbf{\Phi}_{\beta} g\right)(\xi)=-\int_{|\xi|}^{\infty} J_{0}\left(q \sqrt{\tau^{2}-\xi^{2}}\right) g(\tau) d \tau, \xi \in \mathbb{R}, g \in D\left(\mathbf{\Phi}_{\beta}\right) \cap H_{0}^{0} .
$$

Here $J_{\nu}$ is the Bessel function, $\nu=\overline{0, \infty}$. The operator $\boldsymbol{\Phi}_{\beta}$ is invertible, and $\mathbf{\Phi}_{\beta}^{-1}: \mathcal{S}^{\prime} \rightarrow \mathcal{S}^{\prime}, D\left(\boldsymbol{\Phi}_{\beta}^{-1}\right)=R\left(\mathbf{\Phi}_{\beta}\right)=\left\{g \in \mathcal{S}^{\prime} \mid g\right.$ is even and supp $\left.g \subset[-\beta, \beta]\right\}$,

$$
\boldsymbol{\Phi}_{\beta}^{-1} f=\mathcal{F}_{\sigma \rightarrow \xi}^{-1}\left(-i \mu(\mathcal{F} g)\left(\sqrt{\mu^{2}-q^{2}}\right)\right), \quad g \in D\left(\boldsymbol{\Phi}_{\beta}^{-1}\right) .
$$


In particular,

$$
\begin{aligned}
\left(\boldsymbol{\Phi}_{\beta}^{-1} f\right)(\tau) & =f^{\prime}(\tau)+q \tau \int_{|\tau|}^{\infty} \frac{I_{1}\left(q \sqrt{\xi^{2}-\tau^{2}}\right)}{\sqrt{\xi^{2}-\tau^{2}}} f^{\prime}(\xi) d \xi \\
& =-\frac{d}{d \tau} \int_{|\tau|}^{\infty} I_{0}\left(q \sqrt{\xi^{2}-\tau^{2}}\right) f^{\prime}(\xi) d \xi, \tau \in \mathbb{R}, f \in D\left(\boldsymbol{\Phi}_{\beta}^{-1}\right) \cap H_{0}^{0} .
\end{aligned}
$$

Here $I_{\nu}$ is the modified Bessel function, $I_{\nu}(\tau)=i^{-\nu} J_{\nu}(i \tau), \nu=\overline{0, \infty}$. The operators $\boldsymbol{\Phi}_{\beta}$ and $\boldsymbol{\Phi}_{\beta}^{-1}$ are bounded from $H_{0}^{-s}$ to $H_{0}^{-s}, s \geq 0$. Moreover, $\boldsymbol{\Phi}\left(\widetilde{H}_{0}^{-s} \cap D\left(\mathbf{\Phi}_{\beta}\right)\right)=\widetilde{H}_{0}^{-s} \cap D\left(\mathbf{\Phi}_{\beta}\right), s \geq 0$. If $f=\boldsymbol{\Phi}_{\beta} g$, then $f^{\prime} \in L^{\infty}(-\beta, \beta)$ iff $g \in L^{\infty}(-\beta, \beta)$.

Set $W^{0}=\left(\begin{array}{l}W_{0}^{0} \\ W_{1}^{0}\end{array}\right)$ and $Z^{0}=\left(\begin{array}{c}Z_{0}^{0} \\ Z_{1}^{0}\end{array}\right)$. Evidently, $W^{0} \in \mathbb{H}^{1}$ and $Z^{0} \in \widehat{\mathbf{H}}^{1}$.

Throughout the paper the domain and the range of an operator $A$ are denoted by $D(A)$ and $R(A)$, respectively.

\section{Spaces and Operators}

In this section the spaces $\mathbb{H}^{m}, m=\overline{-1,1}$, are investigated and some transformation operators are introduced and studied.

Further throughout the section, we will assume $p=0,1$.

According to (1.4), we have $\sigma(x)=\int_{0}^{x} \frac{d \mu}{\theta^{2}(\mu)}, x \in \mathbb{R}$. Moreover, $\sigma$ is an odd increasing invertible function, and $\sigma(x) \rightarrow+\infty$ as $x \rightarrow+\infty$.

Consider the operator $\mathbf{S}$ introduced and studied in [15]. Let $\mathbf{S}_{p}: H_{0}^{p} \rightarrow \mathbb{H}^{p}$ with the domain $D\left(\mathbf{S}_{p}\right)=H_{0}^{p}$, and

$$
\mathbf{S}_{p} \psi=\frac{\psi \circ \sigma}{\eta}, \quad \psi \in D\left(\mathbf{S}_{p}\right)
$$

where $\psi \circ \sigma$ is the composition of $\psi$ and $\sigma$, i.e., $(\psi \circ \sigma)(x)=\psi(\sigma(x)), x \in \mathbb{R}$. Evidently, $\mathbf{S}_{p}$ is the restriction of $\mathbf{S}_{0}$ to $H_{0}^{p}, p \neq 0$.

By the construction, $\mathbf{S}_{p}$ is invertible, $\mathbf{S}_{p}^{-1}: \mathbb{H}^{p} \rightarrow H_{0}^{p}$ with the domain $D\left(\mathbf{S}_{p}^{-1}\right)=R\left(\mathbf{S}_{p}\right)$, and

$$
\mathbf{S}_{p}^{-1} \varphi=(\eta \varphi) \circ \sigma^{-1}, \quad \varphi \in D\left(\mathbf{S}_{p}^{-1}\right)
$$

In [15], the following theorem is proved.

Theorem 3.1. The following assertions hold:

(i) $\mathcal{D}_{\eta \theta}^{m}\left(\mathbf{S}_{p} \psi\right)=\mathbf{S}_{p}\left(\psi^{(m)}\right), \psi \in H_{0}^{p}, m=\overline{0, p}$; 
(ii) $\llbracket \mathbf{S}_{p} \psi \rrbracket^{p}=\|\psi\|_{0}^{p}, \psi \in H_{0}^{p}, m=\overline{0, p}$;

(iii) $R\left(\mathbf{S}_{p}\right)=\mathbb{H}^{p}$;

(iv) $\mathbf{S}_{p}$ and $\mathbf{S}_{p}^{-1}$ are bounded.

Let us continue $\mathbf{S}_{0}$ to $H_{0}^{-p}$. Introduce the operator $\mathbf{S}_{-p}: H_{0}^{-p} \rightarrow \mathbb{H}^{-p}$ with the domain $D\left(\mathbf{S}_{-p}\right)=H_{0}^{-p}$ such that

$$
\left\langle\left\langle\mathbf{S}_{-p} g, \varphi\right\rangle\right\rangle=\left\langle g, \mathbf{S}_{p}^{-1} \varphi\right\rangle, \quad g \in D\left(\mathbf{S}_{-p}\right), \varphi \in D\left(\mathbf{S}_{p}^{-1}\right) .
$$

This extension $\mathbf{S}_{-p}$ of $\mathbf{S}_{0}$ is also invertible, $\mathbf{S}_{-p}^{-1}: \mathbb{H}^{-p} \rightarrow H_{0}^{-p}$ with the domain $D\left(\mathbf{S}_{-p}^{-1}\right)$, and

$$
\left\langle\mathbf{S}_{-p}^{-1} f, \psi\right\rangle=\left\langle\left\langle f, \mathbf{S}_{p} \psi\right\rangle\right\rangle, \quad f \in D\left(\mathbf{S}_{-p}^{-1}\right), \psi \in D\left(\mathbf{S}_{p}\right) .
$$

Finally, by $\mathbf{S}$, denote the operator $\mathbf{S}_{-1}$ with the domain $D(\mathbf{S})=D\left(\mathbf{S}_{-1}\right)=$ $H_{0}^{-1}$. Evidently, $\mathbf{S}$ is invertible, and $\mathbf{S}^{-1}=\mathbf{S}_{-1}^{-1}: \mathbb{H}^{-1} \rightarrow H_{0}^{-1}$ with the domain $D\left(\mathbf{S}^{-1}\right)=R(\mathbf{S})=\mathbb{H}^{-1}$ (see Theorem 3.1). Taking into account the construction of $\mathbf{S}$ and Theorem 3.1, we obtain

Theorem 3.2. For $m=\overline{-1,1}$, the following assertions hold:

(i) $\mathcal{D}_{\eta \theta}(\mathbf{S} g)=\mathbf{S}\left(g^{\prime}\right), g \in H_{0}^{m}, m \neq-1$;

(ii) $\llbracket \mathbf{S} g \rrbracket^{m}=\|g\|_{0}^{m}, g \in H_{0}^{m}$;

(iii) $\mathbf{S} H_{0}^{m}=\mathbb{H}^{m}$;

(iv) $\mathbf{S} \widehat{H}_{0}^{m}=\widehat{\mathbb{H}}^{m}$;

(v) $\langle\langle f, \varphi\rangle\rangle=\left\langle\mathbf{S}^{-1} f, \mathbf{S}^{-1} \varphi\right\rangle, f \in \mathbb{H}^{-m}, \varphi \in \mathbb{H}^{m}$.

To study control system (2.3), (2.4), we need

Theorem 3.3. $\mathbf{S}(\delta)=\eta(0) \mathcal{D}_{\eta \theta} \delta$.

$\mathrm{P}$ r o o f. Let $\varphi \in \mathbb{H}^{1}$. According to Theorem 3.2, we get $\langle\langle\mathbf{S} \delta, \varphi\rangle\rangle=$ $\left\langle\delta, \mathbf{S}^{-1} \varphi\right\rangle=\eta(0)(\varphi)(0)=\langle\langle\eta(0) \delta, \varphi\rangle\rangle$, which was to be proved.

Due to [15], the following theorem holds.

Theorem 3.4. We have:

(i) $\mathbb{H}^{m} \subset \mathbb{H}^{n}$ is a continuous embedding, $-1 \leq n \leq m \leq 1$;

(ii) $\mathcal{D} \subset \mathbb{H}^{m} \subset \mathcal{D}^{\prime}$ are continuous embeddings, $-1 \leq m \leq 1$;

(iii) $\mathcal{D}$ is dense in $\mathbb{H}^{m}, \mathbb{H}^{m}$ is dense in $\mathbb{H}^{n}, \mathbb{H}^{n}$ is dense in $\mathcal{D}^{\prime},-1 \leq n \leq m \leq 1$. 
In [15], it is also shown that the relation between the Schwartz space $\mathcal{S}$ and the space $\mathbb{H}^{m}$ depends on $\eta$ and $\theta$, i.e., on $k$ and $\rho, m=\overline{-2,2}$.

To study control system $(2.3),(2.4)$, we need the operator transforming each $L^{2}(0,+\infty)$-solution to

$$
-z^{\prime \prime}=\mu^{2} z, \quad \lambda>0
$$

into an $L^{2}(0,+\infty)$-solution to

$$
-y^{\prime \prime}+r y=\mu^{2} y, \quad \lambda>0,
$$

under the boundary condition $\frac{y(\lambda, \mu)}{z(\lambda, \mu)} \rightarrow 1$ as $\lambda \rightarrow+\infty, \mu \in \mathbb{C}, \Re \mu \geq 0$. Here $z^{\prime \prime}$ and $y^{\prime \prime}$ are the derivatives of $z$ and $y$ with respect to $\lambda, \mu$ is a parameter,

$$
\begin{aligned}
r & =\left(\mathcal{D}_{\eta \theta}\left(\theta^{2} \eta^{\prime} / \eta\right)-\widehat{\gamma}-q^{2}\right) \circ \sigma^{-1} \\
& =\left(\frac{1}{4} \sqrt{\frac{k}{\rho}}\left(\sqrt{\frac{k}{\rho}}\left(\frac{k^{\prime}}{k}+\frac{\rho^{\prime}}{\rho}\right)\right)^{\prime}+\left(\frac{1}{4} \sqrt{\frac{k}{\rho}}\left(\frac{k^{\prime}}{k}+\frac{\rho^{\prime}}{\rho}\right)\right)^{2}-\gamma-q^{2}\right) \circ \sigma^{-1} .
\end{aligned}
$$

Taking into account (1.5) and (1.6), we get

$$
r \in L^{\infty}(0,+\infty) \bigcap C^{1}[0,+\infty) \text { and } \lambda r \in L^{1}(0,+\infty) .
$$

It was proved [32, Chap. 3] that this operator is invertible, i.e., there exists an operator transforming each $L^{2}(0,+\infty)$-solution to (3.2) into an $L^{2}(0,+\infty)$ solution to (3.1) under the boundary condition saving the asymptotics of the solutions at infinity.

In [28], this transformation operator and its inverse were extended to $H_{0}^{-1}$. Let us recall the definition for these extensions. We assume (3.4) holds. Let $\Omega=\left\{y=\left(y_{1}, y_{2}\right) \in \mathbb{R}^{2} \mid y_{2}>y_{1}>0\right\}$. Let $K$ be a solution to the system

$$
\begin{cases}K_{y_{1} y_{1}}-K_{y_{2} y_{2}}=r\left(y_{1}\right) K, & y=\left(y_{1}, y_{2}\right) \in \Omega, \\ K\left(y_{1}, y_{1}\right)=\frac{1}{2} \int_{y_{1}}^{\infty} r(\xi) d \xi, & y_{1}>0, \\ \lim _{y_{1}+y_{2} \rightarrow \infty} K_{y_{1}}(y)=\lim _{y_{1}+y_{2} \rightarrow \infty} K_{y_{2}}(y)=0, & y \in \Omega .\end{cases}
$$

Due to [32, Chap. 3], system (3.5) has the unique solution $K$, and $K \in C^{2}(\Omega)$.

Definition 3.1. Denote $\mathbf{T}_{0}: H_{0}^{0} \rightarrow H_{0}^{0}$ with the domain $D\left(\mathbf{T}_{0}\right)=\widehat{H}_{0}^{0}$,

$$
\left(\mathbf{T}_{0} g\right)(\lambda)=g(\lambda)+\lambda \int_{|\lambda|}^{\infty} K(|\lambda|, \xi) g(\xi) d \xi, \quad \lambda \in \mathbb{R}, g \in D\left(\mathbf{T}_{0}\right) .
$$


From [32, Chap. 3], it follows that the operator $\mathbf{T}_{0}$ is invertible, and $\mathbf{T}_{0}^{-1}$ : $H_{0}^{0} \rightarrow H_{0}^{0}, D\left(\mathbf{T}_{0}^{-1}\right)=\widehat{H}_{0}^{0}$,

$$
\left(\mathbf{T}_{0}^{-1} f\right)(\xi)=f(\xi)+\xi \int_{|\xi|}^{\infty} L(|\xi|, \lambda) f(\lambda) d \lambda, \quad \xi \in \mathbb{R}, f \in D\left(\mathbf{T}_{0}^{-1}\right),
$$

where $L \in C^{2}(\Omega)$ is determined by

$$
L(y)+K(y)+\int_{y_{1}}^{y_{2}} L\left(y_{1}, \xi\right) K\left(\xi, y_{2}\right) d \xi=0, \quad y \in \Omega,
$$

or

$$
L(y)+K(y)+\int_{y_{1}}^{y_{2}} K\left(y_{1}, \xi\right) L\left(\xi, y_{2}\right) d \xi=0, \quad y \in \Omega
$$

For the adjoint operators $\mathbf{T}_{0}^{*}$ and $\left(\mathbf{T}_{0}^{-1}\right)^{*}=\left(\mathbf{T}_{0}^{*}\right)^{-1}$ we have $\mathbf{T}_{0}^{*}: H_{0}^{0} \rightarrow H_{0}^{0}$, $D\left(\mathbf{T}_{0}^{*}\right)=\widehat{H}_{0}^{0}=\mathcal{R}\left(\left(\mathbf{T}_{0}^{*}\right)^{-1}\right)$,

$$
\left(\mathbf{T}_{0}^{*} \varphi\right)(\xi)=\varphi(\xi)+\xi \int_{0}^{|\xi|} K(\lambda,|\xi|) \varphi(\lambda) d \lambda, \quad \xi \in \mathbb{R}, \varphi \in D\left(\mathbf{T}_{0}^{*}\right)
$$

and $\left(\mathbf{T}_{0}^{*}\right)^{-1}: H_{0}^{0} \rightarrow H_{0}^{0}, D\left(\left(\mathbf{T}_{0}^{*}\right)^{-1}\right)=\widehat{H}_{0}^{0}=\mathcal{R}\left(\mathbf{T}_{0}^{*}\right)$,

$$
\left.\left(\left(\mathbf{T}_{0}^{*}\right)^{-1} \psi\right)(\lambda)=\psi(\lambda)+\operatorname{sgn} \lambda \int_{0}^{|\lambda|} L(\xi,|\lambda|) \psi(\xi) d \xi, \quad \lambda \in \mathbb{R}, \psi \in D\left(\left(\mathbf{T}_{0}^{*}\right)^{-1}\right)\right) .
$$

Due to [28], the following theorem is valid.

Theorem 3.5. We have:

(i) $\mathbf{T}_{0}^{*}$ is bounded from $H_{0}^{p}$ to $H_{0}^{p}$;

(ii) $\left(\mathbf{T}_{0}^{*}\right)^{-1}$ is bounded from $H_{0}^{p}$ to $H_{0}^{p}$;

(iii) $\mathbf{T}_{0}^{*}\left(\widehat{H}_{0}^{p}\right)=\widehat{H}_{0}^{p}$ and $\left(\mathbf{T}_{0}^{*}\right)^{-1}\left(\widehat{H}_{0}^{p}\right)=\widehat{H}_{0}^{p}$.

Definition 3.2. Denote by $\mathbf{T}_{r}$ the operator $\left(\left.\mathbf{T}_{0}^{*}\right|_{H_{0}^{1}}\right)^{*}$. We have $\mathbf{T}_{r}: H_{0}^{-1} \rightarrow$ $H_{0}^{-1}, D\left(\mathbf{T}_{r}\right)=\widehat{H}_{0}^{-1},\left\langle\mathbf{T}_{r} g, \varphi\right\rangle=\left\langle g, \mathbf{T}_{0}^{*} \varphi\right\rangle, g \in D\left(\mathbf{T}_{r}\right)=\widehat{H}_{0}^{-1}, \varphi \in \widehat{H}_{0}^{1}$. 
Then $\mathbf{T}_{r}^{-1}=\left(\left.\left(\mathbf{T}_{0}^{*}\right)^{-1}\right|_{H_{0}^{1}}\right)^{*}$ and $\mathbf{T}_{r}^{-1}: H_{0}^{-1} \rightarrow H_{0}^{-1}, D\left(\mathbf{T}_{r}^{-1}\right)=\widehat{H}_{0}^{-1}$,

$$
\left\langle\mathbf{T}_{r}^{-1} f, \psi\right\rangle=\left\langle g,\left(\mathbf{T}_{0}^{*}\right)^{-1} \psi\right\rangle, \quad f \in D\left(\mathbf{T}_{r}^{-1}\right)=\widehat{H}_{0}^{-1}, \psi \in H_{0}^{1} .
$$

The following four theorems were proved in [28].

Theorem 3.6. We have

(i) $\mathbf{T}_{r}$ is bounded from $\mathrm{H}_{0}^{-p}$ to $\mathrm{H}_{0}^{-p}$;

(ii) $\mathbf{T}_{r}^{-1}$ is bounded from $H_{0}^{-p}$ to $H_{0}^{-p}$;

(iii) $D\left(\mathbf{T}_{r}\right)=R\left(\mathbf{T}_{r}^{-1}\right)=\widehat{H}_{0}^{-1}=D\left(\mathbf{T}_{r}^{-1}\right)=R\left(\mathbf{T}_{r}\right)$;

(iv) $\mathbf{T}_{r}\left(\widehat{H}_{0}^{-p}\right)=\widehat{H}_{0}^{-p}$ and $\mathbf{T}_{r}^{-1}\left(\widehat{H}_{0}^{-p}\right)=\widehat{H}_{0}^{-p}$.

Theorem 3.7. We have:

$$
\begin{aligned}
\left((d / d \lambda)^{2}-r(|\lambda|)\right)\left(\mathbf{T}_{r} g\right)= & \mathbf{T}_{r}\left((d / d \xi)^{2} g\right)+2 \delta(\lambda) \int_{0}^{\infty} K_{y_{1}}(0, \xi) g(\xi) d \xi \\
& -\delta(\lambda) \int_{0}^{\infty} r(\xi) d \xi g(+0), g \in \widehat{H}_{0}^{1} .
\end{aligned}
$$

Moreover, if $g \in \widehat{H}_{0}^{1}$ and $g^{\prime}(+0)$ exists, then

$$
\left((d / d \lambda)^{2}-r(|\lambda|)\right)\left(\mathbf{T}_{r} g\right)=\mathbf{T}_{r}\left((d / d \xi)^{2} g\right)+2 \delta(\lambda)\left(\left(\mathbf{T}_{r} g\right)^{\prime}(+0)-g^{\prime}(+0)\right) .
$$

Theorem 3.8. We have

$$
\begin{aligned}
(d / d \xi)^{2}\left(\mathbf{T}_{r}^{-1} f\right)= & \mathbf{T}_{r}^{-1}\left(\left((d / d \lambda)^{2}-r(|\lambda|)\right) f\right)+2 \delta(\xi) \int_{0}^{\infty} L_{y_{1}}(0, \lambda) f(\lambda) d \lambda \\
& -\delta(\xi) \int_{0}^{\infty} r(\lambda) d \lambda f^{\prime}(+0) f \in \widehat{H}_{0}^{0} .
\end{aligned}
$$

Moreover, if $f \in \widehat{H}_{0}^{1}$ and $f^{\prime}(+0)$ exists, then

$$
(d / d \xi)^{2}\left(\mathbf{T}_{r}^{-1} f\right)=\mathbf{T}_{r}^{-1}\left(\left((d / d \lambda)^{2}-r(|\lambda|)\right) f\right)+2 \delta(\xi)\left(\left(\mathbf{T}_{r}^{-1} f\right) r^{\prime}(+0)-f^{\prime}(+0)\right) .
$$

Theorem 3.9. $\mathbf{T}_{r}(\delta)=\delta$.

The kernels $K$ and $L$ of the integral operators $\mathbf{T}_{r}$ and $\mathbf{T}_{r}^{-1}$ were found explicitly in [14, Example 5.1] for $r(\lambda)=\beta e^{-\lambda}, \lambda>0$, where $\beta>0$ is a constant. 


\section{Transformations between Solutions to the Main and the Auxiliary Control Systems}

In this section, it is shown that, using the transformation operator $\mathbf{S T}_{r}$ and its inverse, we can transform each solution to auxiliary control system (2.6), (2.7) into a solution to main control system (2.3), (2.4) and vise versa (Theorems 4.2 and 4.4). Note that Theorems 4.2, 4.4 and Corollary 4.3 are analogs of some assertions from [28] for the case $q=0$.

Theorem 4.1. Let $Z$ be a solution to (2.6), (2.7) for some $v \in L^{\infty}(0, T)$ and $Z^{0} \in \widehat{\mathbf{H}}^{1}$. Then (2.8) holds.

P r o o f. $\quad$ Put $\mathbf{J}=\frac{1}{2} J_{0}\left(q \sqrt{t^{2}-\xi^{2}}\right)(H(\xi+t)-H(\xi-t)), \xi \in \mathbb{R}, t \geq 0$. According to [10], we have

$$
\begin{aligned}
Z(\xi, t)= & \mathbf{J}_{t}(\xi, t) * Z_{0}^{0}(\xi)+\mathbf{J}(\xi, t) * Z_{1}^{0}(\xi) \\
& +2 \int_{0}^{t} \mathbf{J}(\xi, t-\tau) v(\tau) d \tau, \quad \xi \in \mathbb{R}, t \geq 0,
\end{aligned}
$$

where $*$ is the convolution with respect to $\xi$. Therefore,

$$
\begin{aligned}
Z_{\xi}(\xi, t)= & \mathbf{J}_{t}(\xi, t) * Z_{0}^{0 \prime}(\xi)+\mathbf{J}(\xi, t) * Z_{1}^{0 \prime}(\xi) \\
& +2 \int_{0}^{t} \mathbf{J}_{\xi}(\xi, t-\tau) v(\tau) d \tau+v(t), \quad \xi \in \mathbb{R}, t \geq 0 .
\end{aligned}
$$

Hence (2.8) holds.

Theorem 4.2. Let $Z$ be a solution to (2.6), (2.7) for some $v \in L^{\infty}(0, T)$ and $Z^{0} \in \widehat{\mathbf{H}}^{1}$. Let $W(\cdot, t)=\mathbf{S T}_{r} Z(\cdot, t), t \in[0, T]$. Then $W$ is a solution to (2.3), (2.4) with $W^{0}=\mathbf{S} \mathbf{T}_{r} Z^{0}$ and

$$
\eta(0) \theta^{2}(0) u(t)=v(t)-R Z^{+}(0, t)+\int_{0}^{\infty} K_{y_{1}}(0, \xi) Z(\xi, t) d \xi, \quad t \in[0, T],
$$

and (2.5) is valid. Here $R=\frac{1}{2} \int_{0}^{\infty} r(\xi) d \xi$, and $(\cdot)^{+}$means the restriction to $[0,+\infty) \times[0, T]$. Moreover,

$$
\begin{aligned}
\| \rrbracket\left(\begin{array}{c}
W(\cdot, t) \\
W_{t}(\cdot, t)
\end{array}\right) \rrbracket \rrbracket & \leq B_{0}\|\|\left(\begin{array}{c}
Z(\cdot, t) \\
Z_{t}(\cdot, t)
\end{array}\right) \|, \quad t \in[0, T], \\
\|u\|_{L^{\infty}(0, T)} & \leq B_{1}\left(\|v\|_{L^{\infty}(0, T)}+(1+T)\|\| Z^{0}\|\|\right),
\end{aligned}
$$

where $B_{0}>0$ and $B_{1}>0$ are some constants independent of $T$. 
P r o o f. Applying Theorem 3.7, we see that $W$ is a solution to (2.3), (2.4) with $W^{0}=\mathbf{S} \mathbf{T}_{R} Z^{0}$ and $U$ determined by (4.3). Let us prove (2.5). Due to Lemma 4.1, (2.8) holds. Taking into account (4.3), we get

$$
\begin{aligned}
W_{x}^{+}(0, t)= & \left(\mathbf{S T}_{R} Z\right)_{x}^{+}(0, t)=\frac{1}{\eta(0) \theta^{2}(0)}\left(Z_{x}^{+}(0, t)\right. \\
& \left.+\int_{0}^{\infty} K_{y_{1}}(0, \xi) Z(\xi, t) d \xi-R Z^{+}(0, t)\right)=u(t), \quad t \in[0, T],
\end{aligned}
$$

i.e., (2.5) holds. According to Theorem 3.6, there exists a constant $B_{0}>0$ such that (4.4) holds. To prove (4.5), we rewrite (4.3). Taking into account (4.1) and setting $\mathbf{G}(\xi, t)=\left(R J_{0}\left(q \sqrt{t^{2}-\xi^{2}}\right)-\int_{\xi}^{t} K_{y_{1}}(\xi, \mu) J_{0}\left(q \sqrt{t^{2}-\mu^{2}}\right) d \mu\right)(H(\xi+t)-$ $H(\xi-t)), \xi \in \mathbb{R}, t \geq 0$, we obtain

$$
\begin{aligned}
\eta(0) \theta^{2}(0) u(t)= & v(t)-\frac{\partial}{\partial t} \int_{0}^{t} \mathbf{G}(\xi, t) Z_{0}^{0}(\xi) d \xi \\
& -\int_{0}^{t} \mathbf{G}(\xi, t) Z_{1}^{0}(\xi) d \xi-\int_{0}^{t} \mathbf{G}(0, t-\tau) v(\tau) d \tau \\
= & v(t)-Z_{0}^{0}(t)-\int_{0}^{t} \mathbf{G}_{t}(\xi, t) Z_{0}^{0}(\xi) d \xi \\
& -\int_{0}^{t} \mathbf{G}(\xi, t) Z_{1}^{0}(\xi) d \xi-\int_{0}^{t} \mathbf{G}(0, t-\tau) v(\tau) d \tau, t \in[0, T] .
\end{aligned}
$$

Since $Z_{0}^{0} \in \widehat{H}_{0}^{1}$, we have

$$
\left|Z_{0}^{0}(t)\right| \leq \int_{-\infty}^{\infty} \frac{1}{\sqrt{1+\sigma^{2}}}\left|\sqrt{1+\sigma^{2}}\left(\mathcal{F} Z_{0}^{0}\right)(\sigma)\right|^{2} d \sigma \leq \pi\left\|Z_{0}^{0}\right\|_{0}^{1},
$$

where $\mathcal{F}$ is the Fourier transform operator. Then, using the estimates given in [32, Chap. 3] for the kernel $K$ and its derivatives, we get

$$
|\mathbf{G}(\xi, t)| \leq A_{0} \quad \text { and } \quad\left|\mathbf{G}_{y}(\xi, t)\right| \leq A_{1} t, \quad t \geq \xi \geq 0,
$$

where $A_{0}>0$ and $A_{1}>0$ are constants. Summarizing (4.6)-(4.8), we conclude that (4.5) is true. The theorem is proved. 
Corollary 4.3. If $W$ is a solution to (2.3), (2.4) for some $u \in L^{\infty}(0, T)$ and $W^{0} \in \mathbb{H}^{1}$, then (2.5) holds.

P r o o f. Put $Z(\cdot, t)=\mathbf{T}_{r}^{-1} \mathbf{S}^{-1} W(\cdot, t), t \in[0, T]$. Applying Theorem 3.8, we see that $Z$ is a solution to $(2.6),(2.7)$ with $Z^{0}=\mathbf{T}_{r}^{-1} \mathbf{S}^{-1} W^{0}$ and

$$
\begin{aligned}
v(t)= & \eta(0) \theta^{2}(0) u(t)+R \eta(0) W^{+}(0, t) \\
& +\int_{0}^{\infty} L_{y_{1}}(0, \lambda)\left(\mathbf{S}^{-1} W(\cdot, t)\right)(\lambda) d \lambda, \quad t \in[0, T] .
\end{aligned}
$$

Here $R=\frac{1}{2} \int_{0}^{\infty} r(\xi) d \xi$, and $(\cdot)^{+}$means the restriction to $[0,+\infty) \times[0, T]$. Substituting $\mathbf{T}_{r} Z(\cdot, t)$ for $W(\cdot, t)$ and taking into account (4.1), we get

$$
\begin{aligned}
v(t)= & \eta(0) \theta^{2}(0) u(t)+\frac{\partial}{\partial t} \int_{0}^{t} \mathbf{G}(\xi, t) Z_{0}^{0}(\xi) d \xi \\
& +\int_{0}^{t} \mathbf{G}(\xi, t) Z_{1}^{0}(\xi) d \xi+\int_{0}^{t} \mathbf{G}(0, t-\tau) v(\tau) d \tau, \quad t \in[0, T] .
\end{aligned}
$$

Theorem 4.2 and (4.6) imply $W_{x}^{+}(0, t)=\widetilde{u}(t), t \in[0, T]$, for

$$
\begin{aligned}
\eta(0) \theta^{2}(0) \widetilde{u}(t)= & v(t)-\frac{\partial}{\partial t} \int_{0}^{t} \mathbf{G}(\xi, t) Z_{0}^{0}(\xi) d \xi \\
& -\int_{0}^{t} \mathbf{G}(\xi, t) Z_{1}^{0}(\xi) d \xi-\int_{0}^{t} \mathbf{G}(0, t-\tau) v(\tau) d \tau, \quad t \in[0, T] .
\end{aligned}
$$

Therefore, $\widetilde{u}(t)=u$ and that was to be proved.

By analogy with Theorem 4.2, we obtain

Theorem 4.4. Let $W$ be a solution to (2.3), (2.4) for some $u \in L^{\infty}(0, T)$ and $W^{0} \in \mathbb{H}^{1}$. Let $Z(\cdot, t)=\mathbf{T}_{r}^{-1} \mathbf{S}^{-1} W(\cdot, t), t \in[0, T]$. Then $Z$ is a solution to (2.6), (2.7) with $Z^{0}=\mathbf{T}_{r}^{-1} \mathbf{S}^{-1} W^{0}$ and

$$
\begin{aligned}
v(t)= & \eta(0) \theta^{2}(0) u(t)+R \eta(0) W^{+}(0, t) \\
& +\int_{0}^{\infty} L_{y_{1}}(0, x) \mathbf{S}^{-1} W(x, t) d x, \quad t \in[0, T] .
\end{aligned}
$$


Here $R=\frac{1}{2} \int_{0}^{\infty} r(\xi) d \xi$, and $(\cdot)^{+}$means the restriction to $[0,+\infty) \times[0, T]$. Moreover,

$$
\begin{aligned}
\|\|\left(\begin{array}{c}
Z(\cdot, t) \\
Z_{t}(\cdot, t)
\end{array}\right) \| & \leq C_{0} \llbracket \llbracket\left(\begin{array}{c}
W(\cdot, t) \\
W_{t}(\cdot, t)
\end{array}\right) \rrbracket \rrbracket, \quad t \in[0, T], \\
\|v\|_{L^{\infty}(0, T)} & \leq C_{1} e^{T C_{2}}\left(\|u\|_{L^{\infty}(0, T)}+(1+T) \square\left[W^{0} \square\right]\right),
\end{aligned}
$$

where $C_{0}>0, C_{1}>0$, and $C_{2}>0$ are constants independent of $T$.

$\mathrm{P}$ r o o f. By analogy with (4.3) and (4.4), using Theorem 3.8 instead of Theorem 3.6, we obtain (4.11) and (4.12). Let us prove (4.13). Taking into account (4.10), we get

$$
v(t)=F_{1}(t)+\int_{0}^{t} F_{2}(t-\tau) v(\tau) d \tau, \quad t \in[0, T],
$$

where

$$
\begin{gathered}
F_{1}(t)=\eta(0) \theta^{2}(0) u(t)+\frac{\partial}{\partial t} \int_{0}^{t} \mathbf{G}(\xi, t) Z_{0}^{0}(\xi) d \xi+\int_{0}^{t} \mathbf{G}(\xi, t) Z_{1}^{0}(\xi) d \xi \\
=\eta(0) \theta^{2}(0) u(t)+Z_{0}^{0}(t)+\int_{0}^{t} \mathbf{G}_{t}(\xi, t) Z_{0}^{0}(\xi) d \xi \\
+\int_{0}^{t} \mathbf{G}(\xi, t) Z_{1}^{0}(\xi) d \xi, \quad t \in[0, T], \\
F_{2}(t)=\mathbf{G}(0, t), \quad t \in[0, T] .
\end{gathered}
$$

According to (4.7) and (4.8), we obtain

$$
\begin{aligned}
& \left\|F_{1}\right\|_{L^{\infty}(0, T)} \leq C_{1}\left(\|u\|_{L^{\infty}(0, T)}+(1+T)\|\| Z^{0}\|\|\right), \\
& \left\|F_{2}\right\|_{L^{\infty}(0, T)} \leq C_{2} .
\end{aligned}
$$

Taking into account (4.16) and the generalized Gronwall theorem, we can come to the conclusion that the integral equation

$$
v(t)=\int_{0}^{t} F_{2}(t-\tau) v(\tau) d \tau, \quad t \in[0, T]
$$


has only the trivial solution in $L^{2}(0, T)$. Due to the Fredholm alternative, Eq. (4.14) has the unique solution in $L^{2}(0, T)$. Using again the generalized Gronwall theorem, we obtain

$$
|v(t)| \leq\left\|F_{1}\right\|_{L^{\infty}(0, T)} e^{t\left\|F_{2}\right\|_{L^{\infty}(0,+\infty)}}, \quad t \in[0, T] .
$$

Taking into account (4.16), (4.15), and (4.12), we obtain (4.13). The theorem is proved.

R e m a r k 4.1. Due to [10, Theorem 3.2], system (2.6), (2.7) has the unique solution. Therefore Theorems 4.2, 4.4 yield the uniqueness of the solution to system $(2.3),(2.4)$.

Thus main control system (2.3), (2.4) is the transformation of auxiliary control system (2.6), (2.7) by $\mathbf{S T}_{r}$ and vise versa: control system (2.6), (2.7) is the transformation of control system $(2.3),(2.4)$ by $\mathbf{T}_{r}^{-1} \mathbf{S}^{-1}$.

\section{Conditions for Controllability}

For given $T>0$ and $W^{0} \in \mathbb{H} \mathbb{I}^{1}\left(Z^{0} \in \widehat{\mathbf{H}}^{1}\right)$, denote by $\mathcal{R}_{T}^{g}\left(W^{0}\right)\left(\mathcal{R}_{T}^{s}\left(Z^{0}\right)\right.$, respectively) the set of the states $W^{T} \in \mathbb{H}^{1}\left(Z^{T} \in \widehat{\mathbf{H}}^{1}\right.$, respectively) for which there exists a control $u \in L^{\infty}(0, T)\left(v \in L^{\infty}(0, T)\right.$, respectively) such that problem (2.3)-(2.4) ((2.6)-(2.7), respectively) has the unique solution $W$ ( $Z$, respectively) and $\left(\begin{array}{c}W(\cdot, T) \\ W_{t}(\cdot, T)\end{array}\right)=W^{T}\left(\left(\begin{array}{c}Z(\cdot, T) \\ Z_{t}(\cdot, T)\end{array}\right)=Z^{T}\right.$, respectively $)$.

Definition 5.1. A state $W^{0} \in \mathbb{H} \mathbb{I}^{1}\left(Z^{0} \in \widehat{\mathbf{H}}^{1}\right)$ is called $L^{\infty}$-controllable with respect to control system $(2.3),(2.4)$ ((2.6), (2.7), respectively) at a given time $T>0$ if 0 belongs to $\mathcal{R}_{T}^{g}\left(W^{0}\right)\left(\mathcal{R}_{T}^{s}\left(Z^{0}\right)\right.$, respectively) and approximately $L^{\infty}$ controllable with respect to control system (2.3), (2.4) ((2.6), (2.7), respectively) at a given time $T>0$ if 0 belongs to the closure of $\mathcal{R}_{T}^{g}\left(W^{0}\right)$ in $\mathbb{H}^{1}\left(\mathcal{R}_{T}^{s}\left(Z^{0}\right)\right.$ in $\widehat{\mathbf{H}}^{1}$, respectively).

For a given $W^{0} \in \mathbb{H I}^{0}\left(Z^{0} \in \widehat{\mathbf{H}}^{0}\right)$, denote $\mathcal{R}^{g}\left(W^{0}\right)=\bigcup_{T>0} \mathcal{R}_{T}^{g}\left(W^{0}\right)\left(\mathcal{R}^{s}\left(Z^{0}\right)=\right.$ $\bigcup_{T>0} \mathcal{R}_{T}^{s}\left(Z^{0}\right)$, respectively).

Definition 5.2. A state $W^{0} \in \mathbb{H}^{1}\left(Z^{0} \in \widehat{\mathbf{H}}^{1}\right)$ is called approximately $L^{\infty}$ controllable with respect to control system (2.3), (2.4) ((2.6), (2.7), respectively) at a free time if 0 belongs to the closure of $\mathcal{R}^{g}\left(W^{0}\right)$ in $\mathbb{I} \mathbb{I}^{1}\left(\mathcal{R}^{s}\left(Z^{0}\right)\right.$ in $\widehat{\mathbf{H}}^{1}$, respectively).

Theorems 4.2 and 4.4 imply

Corollary 5.1. Let $W^{0} \in \mathbb{H} \mathbb{I}^{1}, Z^{0}=\mathbf{S T}_{R} W^{0}$, and a time $T>0$ be given. Then 
(i) $W^{0}$ is approximately $L^{\infty}$-controllable with respect to system $(2.3),(2.4)$ at the time $T$ iff $Z^{0}$ is approximately $L^{\infty}$-controllable with respect to system (2.6), (2.7) at the same time;

(ii) $W^{0}$ is $L^{\infty}$-controllable with respect to system (2.3), (2.4) at the time $T$ iff $Z^{0}$ is $L^{\infty}$-controllable with respect to system (2.6), (2.7) at the same time.

Due to $[11,12,14]$, we have the following three theorems.

Theorem 5.2. Let $Z^{0} \in \widehat{\mathbf{H}}^{1}$ and a time $T>0$ be given. Then

(i) $Z^{0}$ is approximately $L^{\infty}$-controllable with respect to control system $(2.6),(2.7)$ at the time $T$ iff

$$
\begin{aligned}
& \operatorname{supp} Z_{0}^{0} \subset[-T, T] \\
& Z_{1}^{0}-\boldsymbol{\Phi}_{T}\left(\operatorname{sgn} \xi\left(\boldsymbol{\Phi}_{T}^{-1} Z_{0}^{0}\right)^{\prime}\right)=0
\end{aligned}
$$

(ii) $Z^{0}$ is $L^{\infty}$-controllable with respect to control system (2.6), (2.7) at the time $T$ iff $Z_{1}^{0} \in L^{\infty}(0, T)$ and (5.1), (5.2) hold.

Theorem 5.3. Let $q=0$. A state $Z^{0} \in \widehat{\mathbf{H}}^{1}$ is approximately $L^{\infty}$-controllable with respect to control system (2.6), (2.7) at a free time iff

$$
Z_{1}^{0}-\operatorname{sgn} \xi Z_{0}^{0 \prime}=0 \text {. }
$$

Theorem 5.4. Let $q>0$. Each state $Z^{0} \in \widehat{\mathbf{H}}^{1}$ is approximately $L^{\infty}$ controllable with respect to control system (2.6), (2.7) at a free time.

Note that if $q=0$, then (5.2) is equivalent to (5.3).

Corollary 5.1 and Theorem 5.2 imply

Theorem 5.5. Let $W^{0} \in \mathbb{H} \mathbb{I}^{1}$ and a time $T>0$ be given. Then

(i) $W^{0}$ is approximately $L^{\infty}$-controllable with respect to system $(2.3),(2.4)$ at the time $T$ iff

$$
\begin{aligned}
& \operatorname{supp} W_{0}^{0} \subset\left[-\sigma^{-1}(T), \sigma^{-1}(T)\right], \\
& W_{1}^{0}-\left(\mathbf{S T}_{r} \boldsymbol{\Phi}_{T}\right)\left(\operatorname{sgn} x\left(\left(\mathbf{S T}_{r} \boldsymbol{\Phi}_{T}\right)^{-1} W_{0}^{0}\right)^{\prime}\right)=0 ;
\end{aligned}
$$

(ii) $W^{0}$ is $L^{\infty}$-controllable with respect to system (2.3), (2.4) at the time $T$ iff $W_{1}^{0} \in L^{\infty}(0, T)$ and (5.4), (5.5) hold. 
P r o o f. (i) Put $Z^{0}=\mathbf{T}_{r}^{-1} \mathbf{S}^{-1} W^{0}$. According to Corollary 5.1 and Theorem $5.2, W^{0}$ is approximately $L^{\infty}$-controllable with respect to system $(2.3),(2.4)$ at the time $T$ iff conditions (5.1), (5.2) are valid. Evidently, (5.2) is equivalent to (5.5). Taking into account the construction of $\mathbf{S} \mathbf{T}_{r}$ and $\left(\mathbf{S} \mathbf{T}_{r}\right)^{-1}$, we see that (5.1) is equivalent to (5.4)

(ii) Taking into account Corollary 5.1, Theorem 5.2, and (i), it is sufficient to show that

$$
W_{1}^{0} \in L^{\infty}(0, T) \quad \text { iff } \quad Z_{1}^{0} \in L^{\infty}(0, T)
$$

for proving (ii). Using the estimate given in [32, Chap. 3] for the kernel $K$, we get

$$
\begin{aligned}
\left\|W_{1}^{0}\right\|_{L^{\infty}(0, T)} & \leq\left\|Z_{1}^{0}\right\|_{L^{\infty}(0, T)} \max _{\lambda \in[0, T]}\left|\frac{1}{\eta(\lambda)}\left(1+\int_{0}^{\infty}|K(\lambda, \xi)|^{2} d \xi\right)\right| \\
& \leq C\left\|Z_{1}^{0}\right\|_{L^{\infty}(0, T)},
\end{aligned}
$$

i.e., if $Z_{1}^{0} \in L^{\infty}(0, T)$, then $W_{1}^{0} \in L^{\infty}(0, T)$. The converse assertion can be obtained analogously by using the estimate given in for the kernel $L$ instead of that given for the kernel $K$ [32, Chap. 3]. Hence (5.6) holds, which was to be proved.

Note that if $q=0$, then (5.5) is equivalent to

$$
W_{1}^{0}-\mathbf{S T}_{r}\left(\operatorname{sgn} \xi\left(\mathbf{T}_{r}^{-1} \mathbf{S}^{-1} W_{0}^{0}\right)^{\prime}\right)=0 .
$$

Theorems 4.2 and 4.4 imply

Corollary 5.6. Let $W^{0} \in \mathbb{H}^{1}$ and $Z^{0}=\mathbf{T}_{R} W^{0}$. Then $W^{0}$ is approximately $L^{\infty}$-controllable with respect to control system (2.3), (2.4) at a free time iff $Z^{0}$ is approximately $L^{\infty}$-controllable with respect to control system (2.6), (2.7) at a free time.

Theorems 5.3, 5.4 and Corollary 5.6 yield the following two theorems

Theorem 5.7. Let $q=0$. A state $W^{0} \in \mathbb{H}^{1}$ is approximately $L^{\infty}$-controllable with respect to control system (2.3), (2.4) at a free time iff (5.7) holds.

Theorem 5.8. Let $q>0$. Each state $W^{0} \in \mathbb{H}^{1}$ is approximately $L^{\infty}$ controllable with respect to control system (2.3), (2.4) at a free time.

Taking into account [12], we give the following remark.

$\mathrm{R}$ e $\mathrm{m}$ a $\mathrm{rk}$ 5.2. Let $W^{0} \in \mathbb{H}^{1}$ and $\operatorname{supp} W^{0} \subset[-\beta, \beta]$, where $\beta>0$. Thus, 
(i) If $q=0$, then the state $W^{0}$ is approximately $L^{\infty}$-controllable with respect to control system (2.3), (2.4) at a free time iff condition (5.5) holds, and under this condition the state $W^{0}$ is approximately $L^{\infty}$-controllable with respect to control system (2.3), (2.4) at a given time $T \geq \beta$;

(ii) If $q>0$, then the state $W^{0}$ is always approximately $L^{\infty}$-controllable with respect to control system $(2.3),(2.4)$ at a free time, but the state $W^{0}$ is approximately $L^{\infty}$-controllable with respect to control system $(2.3),(2.4)$ at a given time $T \geq \beta$ iff condition (5.5) holds.

Thus, transformed control system (2.3), (2.4) replicates controllability properties of its original control system (2.6), (2.7) and vise versa.

\section{Examples}

In this section, we give the examples illustrating the results of Secs. $2-5$.

$\mathrm{E} \mathrm{x}$ a m p l e 6.1. Let $\alpha>0, \widehat{k}(x)=(1+|x|)\left(1-\tanh ^{2} x\right), \widehat{\rho}(x)=$ $\frac{1}{(1+|x|) \cosh ^{2} x}, \widehat{\gamma}(x)=(1+|x|)^{2}\left(2 \tanh ^{2} x-1\right)-(1+|x|) \tanh |x|-\frac{1}{4(1+|x|)}$,

$$
\begin{aligned}
& W_{0}^{0}(x)=\frac{H\left(\alpha^{2}-x^{2}\right)}{\ln (1+\alpha)} \cosh x \int_{|x|}^{\alpha} I_{0}\left(\sqrt{\frac{\sqrt{1+\mu}-\sqrt{1+|x|}}{\sqrt{1+\mu}+\sqrt{1+|x|}}}\right) \frac{d \mu}{1+\mu}, \\
& W_{1}^{0}(x)=\frac{H\left(\alpha^{2}-x^{2}\right)}{\ln (1+\alpha)} \cosh x I_{0}\left(\sqrt{\frac{\sqrt{1+\alpha}-\sqrt{1+|x|}}{\sqrt{1+\alpha}+\sqrt{1+|x|}}}\right), \quad x \in \mathbb{R} .
\end{aligned}
$$

Evidently, $\eta(x)=1 / \cosh x, \theta=\sqrt{1+|x|}, \sigma(x)=\operatorname{sgn} x \ln (1+|x|), x \in \mathbb{R}$, $\sigma^{-1}(\lambda)=\operatorname{sgn} \lambda\left(e^{|\lambda|}-1\right), \lambda \in \mathbb{R}$. Therefore, $\varphi \in \mathbb{H}^{0}$ iff $\frac{\varphi}{\sqrt{1+|x|} \cosh x} \in H_{0}^{0}$; $\varphi \in \mathbb{H}^{1}$ iff $\sqrt{1+|x|}\left(\frac{\varphi}{\cosh x}\right)^{\prime} \in H_{0}^{0}$. Hence, $W_{0}^{0} \in \mathbb{H}^{1}$. We have

$$
\begin{aligned}
\nu(x) & =\left(\theta^{2}\left(\theta^{2} \frac{\eta^{\prime}}{\eta}\right)^{\prime}\right)(x)+\left(\theta^{2} \frac{\eta^{\prime}}{\eta}\right)^{2}(x) \\
& =(1+|x|)^{2}\left(2 \tanh ^{2} x-1\right)-(1+|x|) \tanh |x|=\widehat{\gamma}(x)+\frac{1}{4(1+|x|)}, x \in \mathbb{R} .
\end{aligned}
$$

Hence conditions (1.5) and (1.6) hold for $q=0, r(\lambda)=e^{-|\lambda|} / 4, \lambda \in \mathbb{R}$ (see (3.4)).

Let us transform control system $(2.3),(2.4)$ with the given $\widehat{k}, \widehat{\rho}, \widehat{\gamma}, W^{0}$ and some $T>0$ into control system (2.6), (2.7). Put $Z^{0}=\mathbf{T}_{r}^{-1} \mathbf{S}^{-1} W^{0}, Z(\cdot, t)=$ $\mathbf{T}_{r}^{-1} \mathbf{S}^{-1} W^{0}(\cdot, t), t \in[0, T]$. For this $r$, the kernels $K$ and $L$ of the operators $\mathbf{T}_{r}$ 
and $\mathbf{T}_{r}^{-1}$ were calculated in [14, Example 5.1]:

$$
\begin{array}{ll}
K(y)=\widetilde{K}_{y_{2}}(y) \text { and } \quad L(y)=-\widetilde{L}_{y_{1}}(y), & y_{2} \geq y_{1} \geq 0, \\
\widetilde{K}(y)=I_{0}\left(\sqrt{e^{-\frac{y_{1}}{2}}\left(e^{-\frac{y_{1}}{2}}-e^{-\frac{y_{2}}{2}}\right)}\right), & y_{2} \geq y_{1} \geq 0, \\
\widetilde{L}(y)=J_{0}\left(\sqrt{e^{-\frac{y_{2}}{2}}\left(e^{-\frac{y_{1}}{2}}-e^{-\frac{y_{2}}{2}}\right)}\right), & y_{2} \geq y_{1} \geq 0 .
\end{array}
$$

Setting $A=\ln (1+\alpha)$, we obtain

$$
\left(\mathbf{S}^{-1} W_{0}^{0}\right)(\lambda)=\frac{1}{A} H\left(A^{2}-\lambda^{2}\right) \int_{|\lambda|}^{A} \widetilde{K}(|\lambda|, \mu) d \mu, \quad \lambda \in \mathbb{R} .
$$

Therefore, for $\xi \in \mathbb{R}$, we have

$$
\begin{aligned}
Z_{0}^{0}(\xi) & =\left(\mathbf{T}_{r}^{-1} \mathbf{S}^{-1} W_{0}^{0}\right)(\xi) \\
& =\frac{1}{A} H\left(A^{2}-\lambda^{2}\right)\left(\int_{|\xi|}^{A} \widetilde{K}(|\xi|, \mu) d \mu+\int_{|\xi|}^{A} L(|\xi|, \lambda) \int_{\lambda}^{A} \widetilde{K}(\lambda, \mu) d \mu d \lambda\right) \\
& =\frac{1}{A} H\left(A^{2}-\lambda^{2}\right) \int_{|\xi|}^{A}\left(\widetilde{K}(|\xi|, \mu)+\int_{|\xi|}^{\mu} L(|\xi|, \lambda) \widetilde{K}(\lambda, \mu) d \lambda\right) d \mu .
\end{aligned}
$$

Let us evaluate

$$
B(\xi, \mu)=\widetilde{K}(\xi, \mu)+\int_{\xi}^{\mu} L(\xi, \lambda) \widetilde{K}(\lambda, \mu) d \lambda, \quad \mu \geq \xi \geq 0 .
$$

Since $K$ and $L$ are the kernels of $\mathbf{T}_{r}$ and $\mathbf{T}_{r}^{-1}$, respectively, we get

$$
K(\xi, \mu)+L(\xi, \mu)+\int_{\xi}^{\mu} L(\xi, \lambda) K(\lambda, \mu) d \lambda=0, \quad \mu \geq \xi \geq 0 .
$$

From here, taking into account (6.1), we obtain $B_{\mu}(\xi, \mu)=0, \mu \geq \xi \geq 0$. Since $B(\xi, \xi)=\widetilde{K}(\xi, \xi)=1, \xi \geq 0$, we have $B(\xi, \mu)=1, \mu \geq \xi \geq 0$. Then, according to $(6.4)$, we obtain

$$
Z_{0}^{0}(\xi)=\frac{1}{A} H\left(A^{2}-\lambda^{2}\right) \int_{|\xi|}^{A} d \mu=\frac{A-|\xi|}{A} H\left(A^{2}-\lambda^{2}\right), \quad \xi \in \mathbb{R} .
$$


We also have

$$
\begin{aligned}
& \left(\mathbf{S}^{-1} W_{0}^{0}\right)(\lambda)=\frac{1}{A} H\left(A^{2}-\lambda^{2}\right) \widetilde{K}(|\lambda|, A), \quad \lambda \in \mathbb{R}, \\
& Z_{0}^{0}(\xi)=\left(\mathbf{T}_{r}^{-1} \mathbf{S}^{-1} W_{0}^{0}\right)(\xi)=\frac{1}{A} H\left(A^{2}-\lambda^{2}\right) B(|\xi|, A)=\frac{1}{A} H\left(A^{2}-\lambda^{2}\right), \quad \xi \in \mathbb{R} .
\end{aligned}
$$

Since condition (5.3) holds for $Z^{0}$, applying Theorem 5.2, we conclude that $Z^{0}$ is $L^{\infty}$-controllable with respect to system $(2.6),(2.7)$ at any time $T \geq A$. Let $T \geq A$. Put $v(t)=\frac{1}{A} H\left(A^{2}-\lambda^{2}\right), t \in[0, T]$, then

$$
Z(\xi, t)=\frac{(A-t)-|\xi|}{A} H\left((A-t)^{2}-\lambda^{2}\right), \quad \xi \in \mathbb{R}, t \in[0, T],
$$

is the unique solution to (2.6), (2.7). Hence, $Z(\xi, T)=Z_{t}(\xi, T)=0$. Therefore the control $v$ solves the $L^{\infty}$-controllability problem with respect to system (2.6), (2.7) at the time $T$ for $Z^{0}$.

Now we can find a control solving the $L^{\infty}$-controllability problem with respect to system $(2.3),(2.4)$ at the time $T$ and the solution to this system for $W^{0}$. For $t \in[0, T]$, put

$$
\begin{aligned}
u(t) & =v(t)-\frac{1}{2} Z^{+}(0, t) \int_{0}^{\infty} r(\xi) d \xi+\int_{0}^{\infty} K_{y_{1}}(0, \xi) Z(\xi, t) d \xi \\
& =\frac{1}{A} H\left(A^{2}-\lambda^{2}\right)\left(1-\frac{1}{8}(A-t)+\int_{0}^{A-t} \widetilde{K}_{y_{1} y_{2}}(0, \xi)((A-t)-\xi) d \xi\right) \\
& =\frac{1}{A} H\left(A^{2}-\lambda^{2}\right)\left(1-\frac{1}{8}(A-t)-\widetilde{K}_{y_{1}}(0,0)(A-t)+\int_{0}^{A-t} \widetilde{K}_{y_{1}}(0, \xi) d \xi\right) \\
& =\frac{1}{A} H\left(A^{2}-\lambda^{2}\right)\left(1+\int_{0}^{A-t} \frac{\left.2 \sqrt{1-I_{1}\left(\sqrt{1-e^{-\frac{\xi}{2}}}\right.}\right)}{2}\left(\frac{1}{2} e^{-\frac{\xi}{2}}-1\right)\right) d \xi \\
& =\frac{1}{A} H\left(A^{2}-\lambda^{2}\right)\left(I_{0}\left(\sqrt{1-\frac{e^{\frac{t}{2}}}{\sqrt{1+\alpha}}}\right)-\widetilde{I}\left(\sqrt{1-\frac{e^{\frac{t}{2}}}{\sqrt{1+\alpha}}}\right)\right),
\end{aligned}
$$

where $\widetilde{I}(p)=2 \int_{0}^{p} \frac{I_{1}(\mu)}{1-\mu^{2}} d \mu$. According to Theorem 4.2, the control $u$ solves the $L^{\infty}$-controllability problem with respect to system $(2.3),(2.4)$ at the time $T$ 
for $W^{0}$. Moreover, we can find the solution to this system explicitly. We have

$$
\begin{aligned}
\left(\mathbf{T}_{r} z(\cdot, t)\right)(\lambda) & =Z(\lambda, t)+\int_{|\lambda|}^{\infty} \widetilde{K}_{y_{2}}(|\lambda|, \xi) Z(\xi, t) d \xi \\
& =\frac{1}{A} H\left((A-t)^{2}-\lambda^{2}\right) \int|\lambda|^{A-t} \widetilde{K}(|\lambda|, \xi) d \xi
\end{aligned}
$$

Therefore,

$$
\begin{aligned}
W(x, t)= & \left(\mathbf{S T}_{r} Z(\cdot, t)\right)(x)=\frac{H\left(\left(e^{A-t}-1\right)^{2}-x^{2}\right)}{A} \int_{\ln (1+|x|)}^{A-t} \widetilde{K}(\ln (1+|x|), \xi) d \xi \\
= & H\left(\left((1+\alpha) e^{-t}-1\right)^{2}-x^{2}\right) \frac{\cosh x}{\ln (1+\alpha)} \\
& \times \int_{|x|}^{(1+\alpha) e^{-t}-1} I_{0}\left(\sqrt{\frac{\sqrt{1+\mu}-\sqrt{1+|x|}}{\sqrt{1+\mu}+\sqrt{1+|x|}}}\right) \frac{d \mu}{1+\mu}, \quad x \in \mathbb{R}, t \in[0, T],
\end{aligned}
$$

is the unique solution to system (2.3), (2.4).

E x a m p l e 6.2. Let $\widehat{k}(x)=\widehat{\rho}(x)=\frac{1}{1+x^{2}}, \widehat{\gamma}(x)=-\frac{2+x^{4}}{\left(1+x^{2}\right)^{2}}, W_{0}^{0}(x)=0$, and $W_{1}^{0}(x)=e^{-|x|} \sqrt{1+x^{2}}, x \in \mathbb{R}$. Evidently, $\eta(x)=\sqrt{\widehat{\rho}(x)}, \theta(x)=1, \sigma(x)=x$, $x \in \mathbb{R}, \sigma^{-1}(\lambda)=\lambda, \lambda \in \mathbb{R}$. Then we have $\frac{\eta}{\theta} \mathcal{D}_{\eta \theta}^{m}=\left(1+x^{2}\right)^{-1 / 2}\left(\frac{d}{d x}\right)^{m}, m=1,2$. Therefore, $\varphi \in \mathbb{H}^{m}$ iff $\varphi \in H_{-1}^{m}, m=-1,0,1$. Hence, $W_{0}^{0} \in \mathbb{H}^{1}$. We have

$$
\begin{aligned}
\nu(x) & =\left(\theta^{2}\left(\theta^{2} \frac{\eta^{\prime}}{\eta}\right)^{\prime}\right)(x)+\left(\theta^{2} \frac{\eta^{\prime}}{\eta}\right)^{2}(x) \\
& =\left(\frac{x}{1+x^{2}}\right)^{\prime}+\left(\frac{x}{1+x^{2}}\right)^{2}=\frac{2 x^{2}-1}{\left(1+x^{2}\right)^{2}}=1+\widehat{\gamma}(x), \quad x \in \mathbb{R} .
\end{aligned}
$$

Thus conditions (1.5) and (1.6) hold for $q=1, r=0$ (see (3.4)). Due to Theorem 5.5, the state $W^{0}$ is not approximately $L^{\infty}$-controllable with respect to system (2.3), (2.4) at any given time $T>0$ because condition (5.4) is not valid for this state. Nevertheless, according to Theorem 5.8, the state $W^{0}$ is approximately $L^{\infty}$-controllable with respect to system $(2.3),(2.4)$ at a free time.

Let us find controls solving the approximate $L^{\infty}$-controllability problem with respect to system (2.3), (2.4) at a free time. Put $Z^{0}=\mathbf{T}_{r}^{-1} \mathbf{S}^{-1} W^{0}=\eta W^{0}=$ $\left(1+x^{2}\right)^{-1 / 2}$. We have $Z_{0}^{0}(\xi)=0$ and $Z_{1}^{0}(\xi)=e^{-|\xi|}, \xi \in \mathbb{R}$. Put $T_{n}=n^{18}$, $n=\overline{1, \infty}$. In [13, Example 6.1], it was proved that the controls

$$
v_{n}(t)=-\frac{8 n^{8}}{t^{2}} \sin \frac{t}{2 n} \sin \frac{t}{2 n^{6}} \sin \left(\sin \frac{t}{2 n}-\frac{t}{2 n^{6}}\right), \quad t \in\left[0, T_{n}\right], n=\overline{1, \infty},
$$


solve the approximate $L^{\infty}$-controllability problem with respect to system $(2.6)$, (2.7) at a free time. From Theorem 4.2, it follows that the controls $u_{n}(t)=v_{n}(t)$, $t \in\left[0, T_{n}\right], n=\overline{1, \infty}$, solve the approximate $L^{\infty}$-controllability problem with respect to system $(2.3),(2.4)$ at a free time.

Note that for each $t \in \mathbb{R}$, we have $\mathcal{U}_{n}(t) \rightarrow \mathcal{U}(t) \equiv t$ as $n \rightarrow \infty$, where $\mathcal{U}_{n}(t)=u_{n}(t) H\left(T_{n}^{2}-t^{2}\right), n=\overline{1, \infty}$. Therefore the sequence $\left\{\mathcal{U}_{n}\right\}_{n=1}^{\infty}$ converges neither in $L^{2}(\mathbb{R})$, nor in $L^{\infty}(\mathbb{R})$.

Ex a m p l e 6.3. Let $\widehat{k}(x)=(1+|x|) \cosh ^{2} x, \widehat{\rho}(x)=\frac{\cosh ^{2} x}{1+|x|}, \widehat{\gamma}(x)=(1+|x|)^{2}+$ $(1+|x|) \tanh |x|-\frac{2+|x|}{4(1+|x|) \cosh x}, W_{0}^{0}(x)=\frac{8}{\cosh x} I_{2}\left(\frac{1}{\sqrt{1+|x|}}\right), W_{1}^{0}(x)=-\frac{\sqrt{3}}{2} W_{0}^{0}(x)$, $x \in \mathbb{R}$. Evidently, $\eta(x)=\cosh x, \theta=\sqrt{1+|x|}, \sigma(x)=\operatorname{sgn} x \ln (1+|x|), x \in \mathbb{R}$, $\sigma^{-1}(\lambda)=\operatorname{sgn} \lambda\left(e^{|\lambda|}-1\right), \lambda \in \mathbb{R}$. Therefore, $\varphi \in \mathbb{H}^{0}$ iff $\frac{\cosh x}{\sqrt{1+|x|}} \varphi \in H_{0}^{0} ; \varphi \in \mathbb{H}^{1}$ iff $\sqrt{1+|x|}(\varphi \cosh x)^{\prime} \in H_{0}^{0}$. Hence, $W_{0}^{0} \in \mathbb{H}^{1}$. We have

$$
\begin{aligned}
\nu(x) & =\left(\theta^{2}\left(\theta^{2} \frac{\eta^{\prime}}{\eta}\right)^{\prime}\right)(x)+\left(\theta^{2} \frac{\eta^{\prime}}{\eta}\right)^{2}(x) \\
& =(1+|x|)^{2}-(1+|x|) \tanh |x|=\widehat{\gamma}(x)+\frac{1}{4}+\frac{1}{4(1+|x|)}, x \in \mathbb{R} .
\end{aligned}
$$

Thus conditions (1.5) and (1.6) hold for $q=1 / 2, r(\lambda)=e^{-|\lambda|} / 4, \lambda \in \mathbb{R}$ (see (3.4)). Due to Theorem 5.5, the state $W^{0}$ is not approximately $L^{\infty}$-controllable with respect to system $(2.3),(2.4)$ at any given time $T>0$ because condition (5.4) is not valid for this state. Nevertheless, according to Theorem 5.8, the state $W^{0}$ is approximately $L^{\infty}$-controllable with respect to system $(2.3),(2.4)$ at a free time.

Let us find controls solving the approximate $L^{\infty}$-controllability problem with respect to system (2.3), (2.4) at a free time. Put $Z^{0}=\mathbf{T}_{r}^{-1} \mathbf{S}^{-1} W^{0}$. For this $r$, the kernels $K$ and $L$ of the operators $\mathbf{T}_{r}$ and $\mathbf{T}_{r}^{-1}$ were calculated in [14, Example 5.1]. In our paper they are given by $(6.1)-(6.3)$. We obtain $\left(\mathbf{S}^{-1} W_{0}^{0}\right)(\lambda)=8 I_{2}\left(e^{-\frac{|\lambda|}{2}}\right)$, $\lambda \in \mathbb{R}$. Setting $a=e^{-\frac{|\xi|}{2}}$ and $b=e^{-\frac{\lambda}{2}}$, we get

$$
\begin{aligned}
& \left(\mathbf{T}_{r}^{-1} \mathbf{S}^{-1} W_{0}^{0}\right)(\xi) \\
& \quad=8 I_{2}\left(e^{-\frac{|\xi|}{2}}\right)-2 \int_{|\xi|}^{\infty} e^{-\frac{|\xi|+\lambda}{2}} \frac{J_{1}\left(\sqrt{e^{-\frac{\lambda}{2}}\left(e^{-\frac{|\xi|}{2}}-e^{-\frac{\lambda}{2}}\right)}\right)}{\sqrt{e^{-\frac{\lambda}{2}}\left(e^{-\frac{|\xi|}{2}}-e^{-\frac{\lambda}{2}}\right)}} I_{2}\left(e^{-\frac{\lambda}{2}}\right) d \lambda \\
& \quad=8 I_{2}(a)-4 a \int_{0}^{a} \frac{J_{1}(\sqrt{b(a-b)})}{\sqrt{b(a-b)}} I_{2}(b) d b
\end{aligned}
$$


Setting $n=k-m$, we get

$$
\begin{aligned}
4 a \int_{0}^{a} & \frac{J_{1}(\sqrt{b(a-b)})}{\sqrt{b(a-b)}} I_{2}(b) d b \\
& =4 a \sum_{m=0}^{\infty} \sum_{m=0}^{\infty} \frac{(-1)^{n}}{n !(n+1) ! m !(m+2) ! 2^{2 m+2 n+3}} \int_{0}^{a}(a-b)^{n} b^{2 m+n+2} d b \\
& =4 a \sum_{m=0}^{\infty} \sum_{n=0}^{\infty} \frac{(-1)^{n} a^{2 m+2 n+3}(2 m+n+2) !}{(n+1) ! m !(m+2) !(2 m+2 n+3) ! 2^{2 m+2 n+3}} \\
& =\sum_{m=0}^{\infty} \sum_{k=m}^{\infty} \frac{(-1){ }^{k+m} a^{2 k+4}(k+m+2) !}{(k-m+1) ! m !(m+2) !(2 k+3) ! 2^{2 k+1}} \\
& =\sum_{k=0}^{\infty} \frac{(-1)^{k} a^{2 k+4}}{(k+1) !(2 k+3) ! 2^{2 k+1}} \sum_{m=0}^{k}(k+1)(-1)^{m} \frac{(k+m+2) !}{(m+2) !} \\
& =\sum_{k=0}^{\infty} \frac{a^{2 k+4}}{(k+1) !(k+3) ! 2^{2 k+1}}
\end{aligned}
$$

because

$$
\begin{aligned}
\sum_{m=0}^{k}\left(\begin{array}{c}
k+1 \\
m
\end{array}\right) & (-1)^{m} \frac{(k+m+2) !}{(m+2) !} \\
& =\left.\left(x^{k+2}(1-x)^{k+1}\right)\right|_{x=1}+(-1)^{k} \frac{(2 k+3) !}{(k+3) !}=(-1)^{k} \frac{(2 k+3) !}{(k+3) !} .
\end{aligned}
$$

Taking into account (6.5), we obtain

$$
\left(\mathbf{T}_{r}^{-1} \mathbf{S}^{-1} W_{0}^{0}\right)(\xi)=8 I_{2}\left(e^{-\frac{|\xi|}{2}}\right)-\sum_{k=0}^{\infty} \frac{a^{2 k+4}}{(k+1) !(k+3) ! 2^{2 k+1}}=a^{2}=e^{-|\xi|} .
$$

Therefore, $Z_{0}^{0}(\xi)=e^{-|\xi|}$ and $Z_{1}^{0}(\xi)=-\frac{\sqrt{3}}{2} e^{-|\xi|}, \xi \in \mathbb{R}$. Hence, $Z(\xi, t)=$ $e^{-\frac{\sqrt{3}}{2} t} e^{-|\xi|}, \xi \in \mathbb{R}, t \in\left[0, T_{n}\right]$, is the unique solution to (2.6), (2.7) with $v=v_{n}$ and $T=T_{n}$. Here $v_{n}(t)=e^{-\frac{\sqrt{3}}{2} t}, t \in\left[0, T_{n}\right],\left\{T_{n}\right\}_{n=1}^{\infty} \subset(0, \infty)$ is an increasing sequence, and $T_{n} \rightarrow \infty$ as $n \rightarrow \infty$. When we have

$$
\left\|\left(\begin{array}{c}
Z\left(\cdot, T_{n}\right) \\
Z_{t}\left(\cdot, T_{n}\right)
\end{array}\right)\right\| \leq e^{-\frac{\sqrt{3}}{2} T_{n}} \rightarrow 0 \quad \text { as } n \rightarrow \infty .
$$

Applying Theorem 4.2, we conclude that $W(\cdot, t)=\mathbf{S T}_{r} Z(\cdot, t), t \in\left[0, T_{n}\right]$, is the 
unique solution to $(2.3),(2.4)$ with $u=u_{n}$ and $T=T_{n}$ where

$$
u_{n}(t)=e^{-\frac{\sqrt{3}}{2} t}\left(-\frac{9}{8}+\int_{0}^{\infty} K_{y_{1}}(0, \xi) e^{-\xi} d \xi\right), \quad t \in\left[0, T_{n}\right]
$$

and

$$
\llbracket \llbracket\left(\begin{array}{l}
W\left(\cdot, T_{n}\right) \\
W_{t}\left(\cdot, T_{n}\right)
\end{array}\right) \rrbracket \llbracket \rightarrow 0 \quad \text { as } n \rightarrow \infty
$$

Thus the controls $u_{n}, n=\overline{1, \infty}$, solve the approximate $L^{\infty}$-controllability problem with respect to system $(2.6),(2.7)$ at a free time.

Finally, let us evaluate $u_{n}, n=\overline{1, \infty}$. Taking into account (6.1)-(6.3), we get

$$
K_{y_{1}}(0, \xi)=\left.\frac{1}{8} e^{-\frac{\xi}{2}}\left(\frac{I_{1}(\mu)}{\mu}+\frac{I_{2}(\mu)}{2}+\frac{I_{2}(\mu)}{\mu^{2}}\right)\right|_{\mu=\sqrt{1-e^{-\frac{\xi}{2}}} .}
$$

Then, setting $a=e^{-\frac{\xi}{2}}$, we obtain

$$
\begin{aligned}
\int_{0}^{\infty} K_{y_{1}}(0, \xi) e^{-\xi} d \xi & =-\left.\frac{1}{4} \int_{0}^{1}\left(\frac{I_{1}(\mu)}{\mu}+\frac{I_{2}(\mu)}{2}+\frac{I_{2}(\mu)}{\mu^{2}}\right)\right|_{\mu=\sqrt{1-a}} a^{2} d a \\
& =-\sum_{m=0}^{\infty} \frac{4(m+1)(m+2)+1}{m !(m+2) ! 2^{2 m+5}} \int_{0}^{1}(1-a)^{m} a^{2} d a \\
& =-\sum_{m=0}^{\infty} \frac{4(m+1)(m+2)+1}{(m+2) !(m+3) ! 2^{2 m+4}} \int_{0}^{1}(1-a)^{m} a^{2} d a \\
& =-2\left(I_{3}(1)+I_{1}(1)\right)+\frac{9}{8} .
\end{aligned}
$$

According to (6.6), we have

$$
u_{n}(t)=-2\left(I_{3}(1)+I_{1}(1)\right) e^{-\frac{\sqrt{3}}{2} t}, \quad t \in\left[0, T_{n}\right], n=\overline{1, \infty} .
$$

\section{Appendix}

Lemma 7.1. Let $f \in \mathcal{H}^{0}, \varphi \in \mathcal{H}^{1}$. If there exists $f(0)$, then

$$
\left\langle\left[\mathcal{D}_{\eta \theta} f, \varphi\right]\right\rangle=-\left\langle\left[f, \mathcal{D}_{\eta \theta} \varphi\right]\right\rangle-(f(0), \varphi(0)) .
$$

P r o o f. Put $F(x)=f(x)$ if $x \geq 0$ and $F(x)=0$ otherwise on $\mathbb{R}$. Then $F \in \mathbb{H}^{0}$. For each $l=\overline{1, \infty}$, set $F_{l}(x)=f(x)$ if $0 \leq x \leq l$ and $F_{l}(x)=0$ otherwise on $\mathbb{R}$. Therefore, $F_{l} \in \mathbb{H}^{0} \cap H_{0}^{0}, l=\overline{1, \infty}$, and

$$
\llbracket F-F_{l} \rrbracket_{0}^{0} \rightarrow 0 \quad \text { as } l \rightarrow \infty .
$$


Let $\psi \in \mathcal{D}, \psi(x) \geq 0$ for $x \in \mathbb{R}, \operatorname{supp} \mu \subset[-1,0], \int_{-\infty}^{\infty} \psi(x) d x=1$. Put $F_{l}^{k}=F_{l} * \psi_{k}$, where $\psi_{k}(x)=k \psi(k x), x \in \mathbb{R}, l=\overline{1, \infty}, *$ is the convolution sign. Hence, $F_{l}^{k} \in \mathbb{H}^{0} \cap C^{\infty}(\mathbb{R})$, supp $F_{l}^{k} \in[-1 / k, l], l, k=\overline{1, \infty}$, and for each $l=\overline{1, \infty}$ we have

$$
\left\|F_{l}-F_{l}^{k}\right\|_{0}^{0} \rightarrow 0 \quad \text { as } k \rightarrow \infty
$$

Therefore, for each $l=\overline{1, \infty}$, we get

$$
\llbracket F_{l}-F_{l}^{k} \rrbracket_{0}^{0} \rightarrow 0 \quad \text { as } k \rightarrow \infty .
$$

Summarizing (7.2) and (7.3), we conclude that there exist increasing sequences $\left\{l_{p}\right\}_{p=1}^{\infty}$ and $\left\{k_{p}\right\}_{p=1}^{\infty}$ such that

$$
\left.\llbracket F-\widehat{F}_{p}\right]_{0}^{0} \rightarrow 0 \quad \text { as } p \rightarrow \infty
$$

where $\widehat{F}_{p}=F_{l_{p}}^{k_{p}}, p=\overline{1, \infty}$. According to (7.4), we obtain

$$
\begin{aligned}
\left.\| \mathcal{D}_{\eta \theta}\left(F-\widehat{F}_{p}\right)\right]^{-1} & \leq\left\|\left(S^{-1}\left(F-\widehat{F}_{p}\right)\right)^{\prime}\right\|_{0}^{-1} \\
& \left.\leq\left\|S^{-1}\left(F-\widehat{F}_{p}\right)\right\|_{0}^{0}=\| F-\widehat{F}_{p}\right]^{0} \rightarrow 0 \quad \text { as } p \rightarrow \infty
\end{aligned}
$$

By $f_{p}$, denote the restriction of $\widehat{F}_{p}$ to $[0,+\infty), p=\overline{1, \infty}$. Then $f_{p} \in \mathcal{H}^{0} \cap C^{\infty}(\mathbb{R})$, $\operatorname{supp} f_{p} \in\left[0, l_{p}\right], p=\overline{1, \infty}$, and

$$
\left\langle\left[\mathcal{D}_{\eta \theta}^{m} f_{p}, \varphi\right]\right\rangle \rightarrow\left\langle\left[\mathcal{D}_{\eta \theta}^{m} f, \varphi\right]\right\rangle \quad \text { as } p \rightarrow \infty, \varphi \in \mathcal{H}^{m}, m=0,1
$$

Taking into account the definition of the value of a distribution at the point $x=0$, we have

$$
\begin{aligned}
f_{p}(0) & =\widehat{F}_{p}(0)=\int_{0}^{l_{p}} f(x) k_{p} \psi\left(k_{p} x\right) d x \\
& =\left\langle f\left(\frac{\cdot}{k_{p}}\right), \psi_{-}\right\rangle \rightarrow\left\langle f(0), \psi_{-}\right\rangle=f(0) \quad \text { as } p \rightarrow \infty
\end{aligned}
$$

where $\psi_{-}(x)=\psi(-x), x \in \mathbb{R}$. 
Let $\varphi \in \mathcal{H}^{1}$. Integrating by parts, we obtain

$$
\begin{aligned}
\left\langle\left[\mathcal{D}_{\eta \theta} f_{p}, \varphi\right]\right\rangle & =\int_{0}^{\infty}\left(\left(S^{-1} f_{p}\right)^{\prime}(x),\left(S^{-1} \varphi\right)(x)\right) d x \\
& =\left.\left(\left(S^{-1} f_{p}\right)(x),\left(S^{-1} \varphi\right)(x)\right)\right|_{0} ^{\infty}-\int_{0}^{\infty}\left(\left(S^{-1} f_{p}\right)(x),\left(S^{-1} \varphi\right)^{\prime}(x)\right) d x \\
& =-(f(0), \varphi(0))-\left\langle\left[f, \mathcal{D}_{\eta \theta} \varphi\right]\right\rangle .
\end{aligned}
$$

Taking into account (7.5) and (7.6), we get (7.1), which was to be proved.

\section{References}

[1] P. Antosik, J. Mikusinski, and R. Sikorski, Theory of Distributions. The Sequential Approach, Elsevier, Amsterdam, 1973.

[2] W.D. Bastos, A. Spezamiglio, and C.A. Raposo, On Exact Boundary Controllability for Linearly Coupled Wave Equations. - J. Math. Anal. Appl. 381 (2011), 557-564.

[3] M.I. Belishev and A.F. Vakulenko, On a Control Problem for the Wave Equation in $\mathbb{R}^{3}$. - Zapiski Nauchnykh Seminarov POMI 332 (2006), 19-37. (Russian) (Engl. transl.: J. Math. Sci. 142 (2007), 2528-2539).

[4] C. Castro, Exact Controllability of the 1-D Wave Equation from a Moving Interior Point. - ESAIM: Control, Optim. Calc. Var. 19 (2013), 301-316.

[5] M. Dreher and I. Witt, Edge Sobolev Spaces and Weakly Hyperbolic Equations. Ann. Mat. Pura Appl. 180 (2002), 451-482.

[6] A. Dutrifoy, S. Schochet, and A.J. Majda, A Simple Justification of the Singular Limit for Equatorial Shallow-Water Dynamics. - Comm. Pure Appl. Math. 62 (2009), 322-333.

[7] J. Eckhardt, Direct and Inverse Spectral Theory of Singular Left-Defnite SturmLiouville Operators. — J. Differ. Eqs. 253 (2012), 604-634.

[8] F. Fanelli and E. Zuazua, Weak Observability Estimates for 1-D Wave Equations with Rough Coeffcients. Ann. Inst. H. Poincaré (C) Non Linear Analysis, doi:10.1016/j.anihpc.2013.10.004

[9] L.V. Fardigola, On Controllability Problems for the Wave Equation on a Half-Plane. — J. Math. Phys., Anal., Geom. 1 (2005), 93-115.

[10] L.V. Fardigola, Controllability Problems for the String Equation on a Half-Axis with a Boundary Control Bounded by a Hard Constant. - SIAM J. Control Optim. 47 (2008), 2179-2199.

[11] L.V. Fardigola, Neumann Boundary Control Problem for the String Equation on a Half-Axis. - Dopovidi Natsionalnoi Akademii Nauk Ukrainy 10 (2009), 36-41. (Ukrainian) 
[12] L.V. Fardigola, Controllability Problems for the 1-d Wave Equation on a Half-Axis with the Dirichlet Boundary Control. - ESAIM: Control, Optim. Calc. Var. 18 (2012), 748-773.

[13] L.V. Fardigola, Controllability Problems for the 1-d Wave Equation on a Half-Axis with the Neumann Boundary Control. - Mathematical Control and Related Fields 3 (2013), 161-183.

[14] L.V. Fardigola, Transformation Operators of the Sturm-Liouville Problem in Controllability Problems for the Wave Equation on a Half-Axis. - SIAM J. Control Optim. 51 (2013), 1781-1801.

[15] L.V. Fardigola, Transformation Operators in Controllability Problems for the Wave Equations with Variable Coefficients on a Half-Axis Controlled by the Diriclet Boundary Condition. - Mathematical Control and Related Fields 5 (2015), 3153.

[16] L.V. Fardigola, Modified Sobolev Spaces in Controllability Problems for the Wave Equation on a Half-Plane. - J. Math. Phys., Anal., Geom. 11 (2015), 18-44.

[17] L.V. Fardigola, Controllability Proplems for the Wave Equation on a Half-Plane and Modified Sobolev Spaces. - Dopovidi Natsionalnoi Akademii Nauk Ukrainy 9 (2019), 18-24. (Ukrainian)

[18] L.V. Fardigola and K.S. Khalina, Controllability Problems for the Wave Equation. — Ukr. Mat. Zh. 59 (2007), 939-952. (Ukrainian) (Engl. transl.: Ukr. Math. J. 59 (2007), 1040-1058.)

[19] G. Floridia, Approximate Controllabilty for Nonlinear Degenerate Parabolic Problems with Bilinear Control. - J. Differ. Eqs. 257 (2014), 3382-3422.

[20] V. Georgiev, S. Lucente, and G. Ziliotti, Decay Estimates for Hyperbolic Systems. - Hokkaido Mathematical Journal 33 (2004), 83-113.

[21] S.G. Gindikin and L.R. Volevich, Distributions and Convolution Equations. Gordon and Breach Sci. Publ., Philadelphia, 1992.

[22] M. Gugat, A. Keimer, and G. Leugering, Optimal Distributed Control of the Wave Equation Subject to State Constraints. - ZAMM Angew. Math. Mech. 89 (2009), 420-444.

[23] M. Gugat and J. Sokolowski, A Note on the Approximation of Dirichlet Boundary Control Problems for the Wave Equation on Curved Domains. - Appl. Analysis 92 (2012), 2200-2214.

[24] M. Jaulent and C. Jean, One-Dimensional Inverse Schrödinger Scattering Problem with Energy-Dependent Potential, I, II. - Ann. Inst. H. Poincaré Sect. A (N.S.) 25 (1976), 105-137.

[25] M. Jaulent and C. Jean, Solution of a Schrödinger Inverse Scattering Problem with a Polynomial Spectral Dependence in the Potemtial. - J. Math. Phys. 23 (1982), $258-266$. 
[26] F.A. Khalilov and E.Ya. Khruslov, Matrix Generalisation of the Modified Kortewegde Vries Equation. - Inverse Problems 6 (1990), 193-204.

[27] K.S. Khalina, Boundary Controllability Problems for the Equation of Oscillation of an Inhomogeneous String on a Half-Axis. - Ukr. Mat. Zh. 64 (2012), 525541.(Ukrainian) (Engl. transl.: Ukr. Math. J. 64 (2012), 594-615.)

[28] K.S. Khalina, On the Neumann Boundary Controllability for a Non-Homogeneous String on a Half-Axis. - J. Math. Phys., Anal., Geom. 8 (2012), 307-335.

[29] K.S. Khalina, On Dirichlet Boundary Controllability for a Non-Homogeneous String on a Half-Axis. - Dopovidi Natsionalnoi Akademii Nauk Ukrainy 10 (2012), 24-29. (Ukrainian)

[30] E.Ya. Khruslov, One-Dimensional Inverse Problems of Electrodynamics. - $Z$ h. Vychisl. Mat. i Mat. Fiz. 25 (1985), 548-561. (Russian)

[31] Y. Lui, Some Sufficient Conditions for the Controllability of the Wave Equation with Variable Coefficients. — Acta Appl. Math. 128 (2013), 181-191.

[32] V.A. Marchenko, Sturm-Liouville Operators and Applications. AMS, Providence, RI, 2011.

[33] Y. Privat, E. Trélat, and E. Zuazua, Optimal Location of Controllers for the OneDimensional Wave Equation. - Ann. Inst. Poincaré Anal Non Linéaire 30 (2013), 1097-1126.

[34] Ch. Seck, G. Bayili, A. Séne et M.T. Niane, Contrôlabilité Exacte de l'Équation des Ondes dans des Espaces de Sobolev non Réguliers pour un Ouvert Polygonal. - Afr. Mat. 23 (2012), 1-9.

[35] M.A. Shubov, Spectral Decomposition Method for Controlled Damped String Reduction of Control Time. - Appl. Analysis 68 (3-8) (1998), 241-259.

[36] G.M. Sklyar and L.V. Fardigola, The Markov Power Moment Problem in Problems of Controllability and Frequency Extinguishing for the Wave Equation on a HalfAxis. - J. Math. Anal. Appl. 267 (2002), 109-134.

[37] E. Zerrik and R. Larhrissi, Regional Target Control of the Wave Equation. International Journal of Systems Science 32 (2001), 1233-1242.

[38] X. Zhang, A Unified Controllability/Observability Theory for Some Stochastic and Deterministic Partial Differential Equations. In: Proceedings of the International Congress of Mathematicians, Vol. IV, Hyderabad, India, 2010, pp. 3008-3034.

[39] X. Zhang and Sh. Zheng, Strichartz Estimates and Local Wellposedness for the Schrödinger Equation with the Twisted Sub-Laplacian. Proceedings of the Centre for Mathematics \& Its Applications at the Australian National University 44, 233243 (2010) http://works.bepress.com/shijun_zheng/23.

[40] E. Zuazua, Controllability and Observability of Partial Differential Equations: Some Results and Open Problems. Handbook of Differential Equations: Evolutionary Equations, Vol. 3. Elsevier Science, 2006. 